\title{
Mechanistic Analysis of an Extracellular Signal-Regulated Kinase 2-Interacting Compound that Inhibits Mutant BRAF-Expressing Melanoma Cells by Inducing Oxidative Stress $\$$
}

\author{
Ramon Martinez, III, ${ }^{1}$ Weiliang Huang, ${ }^{1}$ Ramin Samadani, Bryan Mackowiak, \\ Garrick Centola, Lijia Chen, Ivie L. Conlon, (1)Kellie Hom, Maureen A. Kane, \\ Steven Fletcher, and Paul Shapiro \\ Department of Pharmaceutical Sciences, University of Maryland, Baltimore- School of Pharmacy, Baltimore, Maryland
}

Received August 3, 2020; accepted October 6, 2020

\begin{abstract}
Constitutively active extracellular signal-regulated kinase (ERK) $1 / 2$ signaling promotes cancer cell proliferation and survival. We previously described a class of compounds containing a 1,1dioxido-2,5-dihydrothiophen-3-yl 4-benzenesulfonate scaffold that targeted ERK2 substrate docking sites and selectively inhibited ERK1/2-dependent functions, including activator protein-1-mediated transcription and growth of cancer cells containing active ERK $1 / 2$ due to mutations in Ras G-proteins or BRAF, Proto-oncogene B-RAF (Rapidly Acclerated Fibrosarcoma) kinase. The current study identified chemical features required for biologic activity and global effects on gene and protein levels in A375 melanoma cells containing mutant BRAF (V600E). Saturation transfer difference-NMR and mass spectrometry analyses revealed interactions between a lead compound (SF-3-030) and ERK2, including the formation of a covalent adduct on cysteine 252 that is located near the docking site for ERK/FXF (DEF) motif for substrate recruitment. Cells treated with SF-3-030 showed rapid changes in immediate early gene levels, including DEF motif-containing ERK1/2 substrates in the Fos family. Analysis of transcriptome and proteome changes showed that the SF-3-030 effects overlapped with ATP-competitive or catalytic site inhibitors of MAPK/ERK Kinase
\end{abstract}

1/2 (MEK1/2) or ERK1/2. Like other ERK1/2 pathway inhibitors, SF-3-030 induced reactive oxygen species (ROS) and genes associated with oxidative stress, including nuclear factor erythroid 2-related factor 2 (NRF2). Whereas the addition of the ROS inhibitor $\mathrm{N}$-acetyl cysteine reversed SF-3-030-induced ROS and inhibition of A375 cell proliferation, the addition of NRF2 inhibitors has little effect on cell proliferation. These studies provide mechanistic information on a novel chemical scaffold that selectively regulates ERK1/2-targeted transcription factors and inhibits the proliferation of A375 melanoma cells through a ROS-dependent mechanism.

\section{SIGNIFICANCE STATEMENT}

Constitutive activation of the extracellular signal-regulated kinase (ERK1/2) pathway drives the proliferation and survival of many cancer cell types. Given the diversity of cellular functions regulated by ERK1/2, the current studies have examined the mechanism of a novel chemical scaffold that targets ERK2 near a substrate binding site and inhibits select ERK functions. Using transcriptomic and proteomic analyses, we provide a mechanistic basis for how this class of compounds inhibits melanoma cells containing mutated BRAF and active ERK1/2.

\section{Introduction}

Deregulated protein kinase activity that promotes cell proliferation and survival is a hallmark of many cancers. Thus, targeted inhibition of protein kinases with small molecules has become an important therapeutic strategy to treat cancer $(\mathrm{Wu}$ et al., 2016). The extracellular signal-regulated kinase (ERK) 1

The work was supported by National Institutes of Health National Cancer Institute [Grant R01-CA120215] (to P.S.) and National Institute of General Medical Sciences (NIGMS) [Grant F31-GM100693] (R.S.) and by the University of Maryland Computer-Aided Drug Design Center. In addition, support was provided in part by the University of Maryland-Baltimore, School of Pharmacy Mass Spectrometry Center [SOP1841-IQB2014]. This study made use of NMRbox: National Center for Biomolecular NMR Data Processing and Analysis, a Biomedical Technology Research Resource (BTRR), which is supported by NIH NIGMS [Grant P41-GM111135]. Training support was provided by NIH NIGMS T32 [Grant T32-GM066706] and by NIGMS Initiative for Maximizing Student Development [Grant 2 R25-GM55036].

${ }^{1}$ R.M. and W.H. contributed equally.

https://doi.org/10.1124/jpet.120.000266.

S This article has supplemental material available at jpet.aspetjournals.org. and 2 proteins are members of the mitogen-activated protein kinase family and major regulators of intracellular signaling events that provide cells with a proliferation and survival advantage. Constitutive activation of ERK1/2 occurs through mutations or elevated expression of upstream activators, including receptor tyrosine kinases, Ras G-proteins, and Raf kinases (Reuter et al., 2000; Shapiro, 2002; Bennasroune et al., 2004; Arora and Scholar, 2005; Mendelsohn and Baselga, 2006). Activating mutations in MEK1, a direct activator of the ERK1/2 proteins, may contribute to deregulated ERK1/2 activity (Emery et al., 2009). In addition, activating mutations in a conserved glutamate in a region of ERK2 associated with protein-protein interactions are associated with the progression of cutaneous T-cell lymphomas (Arvind et al., 2005; Mahalingam et al., 2008; da Silva Almeida et al., 2015). A number of small-molecularweight anticancer drugs targeting the ATP binding or catalytic sites on receptor tyrosine kinases, BRAF, and MEK1/2 are being used in the clinic to block ERK1/2 signaling (Flaherty and McArthur, 2010; Zhong and Bowen, 2011; Wu et al., 2015). 
Despite promising initial responses, resistance to these kinase inhibitors is often observed, and subsequent therapeutic options are limited. Drug resistance occurs through several mechanisms, including acquired mutations in the kinase catalytic site targeted by the compounds, activation of alternative mechanisms to turn on proteins downstream of the targeted kinase, or increases in compensatory signaling pathways that provide a survival advantage independent of the kinase being targeted (Poulikakos and Rosen, 2011; Poulikakos and Solit, 2011; Tuma, 2011; Fedorenko et al., 2015). Recent efforts aimed at overcoming inherent or acquired resistance to existing kinase inhibitors are directly targeting ERK1/2 proteins (Morris et al., 2013; Maik-Rachline and Seger, 2016; Germann et al., 2017) and highlight the need to identify alternative approaches to inhibit constitutively active kinase signaling pathways that promote drug resistance and drive the survival of cancer cells.

Using computational modeling and biologic testing, we have previously identified small molecules that are designed to selectively inhibit ERK1/2 signaling by disrupting specific substrate interactions (Hancock et al., 2005; Chen et al., 2006; Boston et al., 2011; Samadani et al., 2015). This approach targeted two defined substrate docking sites on ERK2: the D-recruitment site (DRS) or F-recruitment site (FRS), which interact with substrates containing D-domains or DEF motifs, respectively (Burkhard et al., 2011; Lee et al., 2011; Sammons et al., 2019). Previous studies suggest that most ERK1/2 substrates contain D-domains, although some substrates, such as the ternary complex factor Elk-1, contain both a D-domain and a DEF motif (von Kriegsheim et al., 2009). Several transcription factors that promote cancer cell growth, including c-Myc and Fos family proteins, contain the DEF motif, which is characterized by a phenylalanine-any amino acid-phenylalanine (FXF) sequence that is 6-10 amino acids away from the serine or threonine phosphorylation site. Hydrophobic interactions between the FXF motif and the recruitment site on ERK1/2 facilitate kinase-substrate interactions necessary for efficient phosphoryl transfer. To selectively target DEF motif-containing transcription factors that promote tumor growth, we previously described the identification of a class of compounds containing a 1,1-dioxido-2,5-dihydrothiophen-3-yl 4-benzenesulfonate scaffold that inhibited DEF motif-containing substrates of the activator protein-1 (AP-1) family (Samadani et al., 2015). This class of compounds selectively inhibited the proliferation of melanoma cells harboring BRAF mutations and constitutively active ERK1/2. These compounds were also effective at inhibiting melanoma cell lines that are inherently resistant to clinically relevant inhibitors of mutated BRAF because of elevated mitogen-activated protein kinase kinase kinase- 8 (MAP3K8) (Samadani et al., 2015).

To define the structure-activity relationship for the 1,1dioxido-2,5-dihydrothiophen-3-yl 4-benzenesulfonate-based compounds, the current studies synthesized several analogs and identified a single double bond in the sulfur heterocycle that was essential for the compound's biologic activity. The findings also demonstrate that this class of compounds forms a covalent interaction with ERK2 near the FRS and has differential effects on the expression levels of ERK1/2-regulated transcription factors containing DEF motifs. The most active compound, SF-3-030, was examined for global effects on gene and protein levels to evaluate ERK1/2-dependent and independent mechanisms of action. The findings support a mechanism by which SF-3-030 induced an oxidative stress response that mediated the inhibition of melanoma cells containing mutated and constitutively active BRAF.

\section{Materials and Methods}

Compound Synthesis. All reactions were performed in ovendried glassware under an inert $\left(\mathrm{N}_{2}\right)$ atmosphere. Anhydrous solvents were used as supplied without further purification. Chemicals were purchased from Sigma-Aldrich (St. Louis, MO), Alfa Aesar (Ward Hill, MA), or Oakwood Chemicals (West Columbia, SC). Reactions were monitored by thin-layer chromatography, visualizing with $\mathrm{KMnO}_{4}$ stain and/or UV as appropriate. Silica gel flash column chromatography was performed with $60-\AA$, 230-400 mesh silica gel, and crude reaction mixtures were first adsorbed onto silica gel from $\mathrm{CH}_{2} \mathrm{Cl}_{2}$ at room temperature. ${ }^{1} \mathrm{H}$ and ${ }^{13} \mathrm{C}$ NMR spectra were recorded on a Varian $400-\mathrm{MHz}$ NMR spectrometer (amaZon X; Bruker) at $25^{\circ} \mathrm{C}$. Chemical shifts are reported in parts per million and are referenced to residual nondeuterated solvent peak $\left(\mathrm{CHCl}_{3}: \delta_{\mathrm{H}} 7.26, \delta_{\mathrm{C}} 77.2 ; \mathrm{DMSO}: \delta_{\mathrm{H}} 2.50\right.$, $\delta_{\mathrm{C}}$ 39.5). Prior to biologic testing, final compounds were confirmed to be $>95 \%$ pure by High-performance liquid chromatography. Detailed methods of the chemical synthesis are provided in the Supplemental Data.

Chemicals and Antibodies. The MEK1/2 inhibitor AZD6244 (catalog number BV-2234-5), 6-(4-bromo-2-chloroanilino)-7-fluoro-N(2-hydroxyethoxy)-3-methylbenzimidazole-5-carboxamide), was purchased from Axxora (Farmingdale, NY). The ERK1/2 inhibitor SCH772984 (catalog number S7101), ( $R$ )-1-(2-oxo-2-(4-(4-(pyrimidin-2-yl)phenyl)piperazin-1-yl)ethyl)- $N$-(3-(pyridin-4-yl)-1H-indazol-5-yl)pyrrolidine-3-carboxamide), was purchased from Selleckchem (Houston, TX). Antioxidants $N$-acetyl L-cysteine (NAC) (catalog number A7250), sodium pyruvate (catalog number P2256), and mannitol (catalog number M4125) were purchased from SigmaAldrich. NRF2 inhibitors ML-385 (catalog number SML 1833) and brusatol (catalog number SML 1868) were purchased from SigmaAldrich. Antibodies against total c-Fos (catalog number 2250), FosB/ B2 (catalog number 2251), Fos-related antigen 1 (Fra-1) (catalog number 5281), c-Jun (catalog number 9165), c-Myc (catalog number 5605), Elk-1 (catalog number 9182), $\beta$-actin (catalog number 4970), and cleaved poly (ADP-ribose) polymerase (PARP) (catalog number 9541) were purchased from Cell Signaling Technology (Beverly, MA). The phospho-specific ERK1/2 (pThr183/pTyr185) (catalog number M9692) and $\alpha$-tubulin (catalog number T6074) antibodies were from Sigma-Aldrich. Phosphorylation-specific antibodies against histone H3 (pSer10) (catalog number 9701), Elk-1 (pS383) (catalog number 9186), and MEK1/2 (pSer217/pSer221) (catalog number 9121) were purchased from Cell Signaling Technology. Antibodies against ERK2 (catalog number sc-154), MEK1/2 (catalog number sc-81504), heme

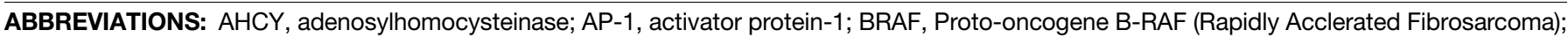

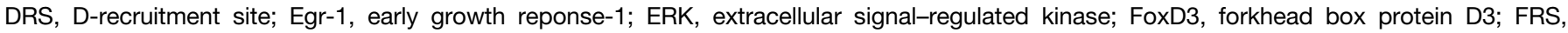

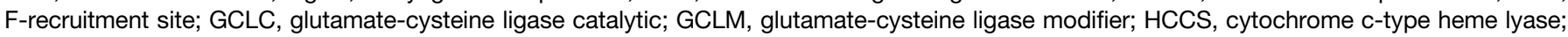

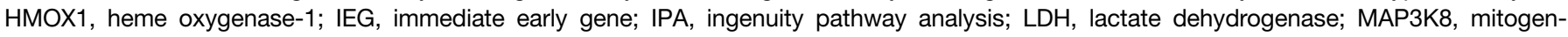

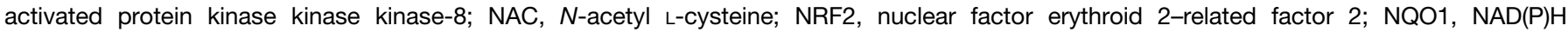

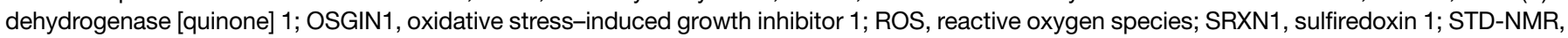
saturation transfer difference-NMR; PARP, poly (ADP-ribose) polymerase; TCF4, transcription factor 4; ZNF774, zinc finger protein 774.
} 
oxygenase-1 (HMOX1) (catalog number sc-136960), and NRF2 (catalog number sc-722) were from Santa Cruz Biotechnology. A second NRF2 antibody (catalog number ab 137550) and the $\mathrm{NAD}(\mathrm{P}) \mathrm{H}$ dehydrogenase [quinone] 1 (NQO1) antibody (catalog number ab34173) were purchased from Abcam (Cambridge, MA). The forkhead box protein D3 (FoxD3) (catalog number 631701) antibody was purchased from BioLegend (San Diego CA). The oxidative stressinduced growth inhibitor 1 (OSGIN1) (catalog number 15248-1-AP), glutamate-cysteine ligase modifier (GCLM) (catalog number 14241-1AP), sulfiredoxin 1 (SRXN1) (catalog number 14273-1-AP), glutamatecysteine ligase catalytic (GCLC) (catalog number 12601-1-AP), aldo-keto reductase family 1 member B10 (catalog number 18252-1-AP), glucose6-phosphate dehydrogenase (catalog number 66373-1-Ig), and thioredoxin reductase 1 antibodies (catalog number 11117-1-AP) were purchased from ProteinTech (Rosemont, IL). The cystine/glutamate transporter antibody (catalog number NB300-317SS) was purchased from Novus Biologicals (Centennial, CO).

Cell Lines. Cell lines used included HeLa cervical carcinoma cells, Jurkat T-cell leukemia cells, and NRas mutated promyelocytic leukemia HL-60 cells. Melanoma cell lines tested included A375 and SK-Mel-28 (homozygous BRAF V600E mutation), SK-MEL-5 (heterozygous BRAF V600E mutation), SK-Mel-2 (BRAF wild type, NRas Q61R mutation), and RPMI-7951 cells (heterozygous BRaf mutation), which are inherently resistant to BRAF inhibitors because of elevated MAP3K8 (Johannessen et al., 2010). Cells were purchased from American Type Culture Collection (Manassas, VA) and grown in Dulbecco's modified Eagle's medium (DMEM)or Eagle's minimal essential medium (EMEM) plus 10\% FBS. All media were supplemented with penicillin and streptomycin. The cell lines have been authenticated by evaluating short tandem repeat DNA profiles and matching with the American Type Culture Collection data base. Cell lines are routinely tested for mycoplasma contamination using the MycoAlert detection kit (Lonza, Walkersville, MD).

Cell Viability/Apoptosis Assays. Cells were seeded at 5000 cells per well in 96-well plates, cultured overnight, and treated for 48 hours with the indicated dose of compounds. $\mathrm{IC}_{50}$ values were determined using nine data points and 3-fold dilutions of $0.001-10 \mu \mathrm{M}$ for AZD6244 and SCH772984 or 0.1-100 $\mu \mathrm{M}$ for SF-3-030. Cell viability was measured according to the manufacturer's instructions using the fluorescent CellTiter Blue Assay (Promega, Madison, WI) or the CellTiter-Glo Luminescent Cell Viability Assay (Promega). To determine the minimal exposure time needed to induce an apoptotic response, cells were exposed to test compounds for various times (0-24 hours), the cells were washed, and then fresh medium was added without test compound for a total of 24 hours. Relative apoptosis was measured by immunoblotting for the cleaved form of PARP.

Reactive Oxygen Species Measurements. Reactive oxygen species (ROS) were measured using the cell-permeable fluorogenic reagent CellROX Deep Red according to the manufacturer's instructions (Thermo Fisher Scientific, Waltham, MA). Briefly, A375 melanoma cells were seeded at $10^{4}$ cells per well in a 96 -well plate. After 24 hours, the cells were coincubated with SF-3-030 \pm various ROS inhibitors as well as $5 \mu \mathrm{M}$ of CellROX Deep Red reagent for 1 hour at $37^{\circ} \mathrm{C}$. Subsequently, cells were washed three times with Dulbecco's modified Eagle's medium containing no Phenol Red. Live cell fluorescence was imaged using a Nikon Eclipse Ti confocal microscope using a $10 \times$ objective magnification. Total fluorescent intensity was obtained in triplicate wells from four frames of view per well ( 500-1000 cells per frame) with a biologic duplicate performed for each treatment condition. The mean \pm S.D. values were determined using NIS-Elements analysis software.

RNA Sequencing Analysis. A375 cells were treated for 1 hour with $10 \mu \mathrm{M}$ AZD6244 or $25 \mu \mathrm{M}$ SF-3-030, and total RNA was isolated (RNAeasy; Qiagen, Hilden, Germany) from treated and untreated cells in duplicate samples. Differential expression analysis was done by GENEWIZ (South Plainfield, NJ) using Illumina Genome Analyzer and HiSeq instruments in the FastQ format as previously described (Anders et al., 2013). Data were analyzed using Ingenuity Pathway
Analysis software. Changes in expression were shown for genes with false discovery rates $<0.05$.

Immunoassays. Immunoblot analysis of protein expression, phosphorylation, and cell proliferation were done as previously described (Samadani et al., 2015). Cells were washed with cold PBS (pH 7.2; Invitrogen, Carlsbad, CA), and protein lysates were collected with $2 \times$ SDS-PAGE sample buffer (4\% SDS, $5.7 \mathrm{M} \beta$-mercaptoethanol, $0.2 \mathrm{M}$ Tris $\mathrm{pH}$ 6.8, 20\% glycerol, $5 \mathrm{mM}$ EDTA). Proteins were separated by SDS-PAGE, analyzed by immunoblotting, and detected using enhanced chemiluminescence (Pierce ECL; Thermo Fisher Scientific). Immunoblots were quantified via densitometry using the Azure c300 Chemiluminescent Western Blot Imaging System (Azure Biosystems, Dublin, CA) for image capture, and data were quantified on the Image Studio Lite Quantification software (v5.2; Li-COR Biotechnology, Lincoln, NE). Immunoassays were also performed using Wes Simple Western (ProteinSimple, San Jose, CA). Electropherograms were quantified using Compass 225 for SW software (v3.1.7; ProteinSimple) applying a Gaussian peak fit distribution for determining area under the curve.

Covalent Modification Analysis by High-Resolution Liquid Chromatography-Tandem Mass Spectrometry. His-tagged ERK2 was purified from Escherichia coli BL21 (DE3) cells transformed with a wild-type erk2 construct using the previously described method (Burkhard et al., 2011). For covalent modification analysis, in vitro kinase reactions containing $100 \mu \mathrm{g}$ of purified ERK2, $1 \mathrm{mM}$ ATP, $1 \times$ NEBuffer for Protein Kinases (New England Biolabs, Ipswich, $\mathrm{MA}$ ), and $50 \mu \mathrm{M} \mathrm{SF-3-030}$ were incubated for 2 hours at $25^{\circ} \mathrm{C}$. After the reactions, ERK2 protein was desalted, reduced, alkylated, and trypsinolyzed on filter as described previously (Wiśniewski et al., 2009; Erde et al., 2014). Tryptic peptides were separated on a nanoACQUITY Ultra Performance Liquid Chromatography (UPLC) analytical column (BEH130 C18, 1.7, $75 \mu \mathrm{m} \times 200 \mathrm{~mm}$; Waters Corporation, Milford, MA) over a 165-minute linear acetonitrile gradient (3\%-40\%) with $0.1 \%$ formic acid on a Waters nanoACQUITY UPLC system (Waters Corporation) and analyzed on a coupled Thermo Scientific Orbitrap Fusion Lumos Tribrid mass spectrometer (Thermo Scientific, San Jose, CA), as described (Williamson et al., 2016). Full scans were acquired at a resolution of 120,000 , and precursors were selected for fragmentation by higher-energy collisional dissociation (normalized collision energy at $32 \%$ ) for a maximum 3 -second cycle. Tandem mass spectra were searched against the ERK2 protein sequence using a SEQUEST HT algorithm (Eng et al., 2008) and an MS Amanda algorithm (Dorfer et al., 2014) with a maximum precursor mass error tolerance of $10 \mathrm{ppm}$. Possible substitution $\left(\mathrm{S}_{\mathrm{N}} 2^{\prime}\right.$ and $\left.\mathrm{S}_{\mathrm{N}} 2,+115.9932\right)$, Michael addition (+324.0126), and carbamidomethylation of cysteine were treated as dynamic modifications. Resulting hits were validated at a maximum false discovery rate of 0.01 using a semisupervised machine learning algorithm, Percolator (Käll et al., 2007). The probabilities of modification sites were computed using a ptmRS algorithm (Taus et al., 2011).

Saturation Transfer Difference-NMR Analysis. Saturation transfer difference-NMR (STD-NMR) analysis of ligand binding to ERK2 was done as previously described for p38 MAPK (Shah et al., 2017). A $1 \mathrm{mM}$ stock solution of SF-3-030 was made in $85 \% \mathrm{D}_{2} \mathrm{O}$ : 15\% d6-DMSO (v/v). STD-NMR samples contained $150 \mathrm{mM} \mathrm{NaCl}$, $50 \mathrm{mM}$ phosphate (pH 7), $200 \mu \mathrm{M}$ SF-3-030, and $5 \mu \mathrm{M}$ ERK2 protein in $\mathrm{D}_{2} \mathrm{O}$. Spectra of both compound and ligand bound protein were recorded on an Agilent DD2 500-MHz spectrometer equipped with a 5 -mm inverse proton-fluorine-carbon-nitrogen probe head at $25^{\circ} \mathrm{C}$. Further detailed methods of the NMR protocol used are provided in the Supplemental Data.

Differential Protein Expression by High-Resolution Liquid Chromatography-Tandem Mass Spectrometry. A375 cells grown on $10-\mathrm{cm}$ plates were treated for 4 and 12 hours with $0.1 \%$ DMSO vehicle, $25 \mu \mathrm{M}$ SF-3-030, or $10 \mu \mathrm{M}$ SCH772984. After one wash in cold PBS, the cells were collected by scraping twice with cold PBS and centrifuged at 3000 rpm for 2 minutes; the cell pellets 
were stored at $-80^{\circ} \mathrm{C}$. Cells were lysed in $4 \%$ sodium deoxycholate, reduced, alkylated, and trypsinolyzed on filter as described (Wiśniewski et al., 2009). Tryptic peptides were separated on a nanoACQUITY UPLC analytical column (CSH130 C18, $1.7 \mu \mathrm{m}, 75 \mu \mathrm{m} \times 200 \mathrm{~mm}$; Waters Corporation) over a 180-minute linear acetonitrile gradient (3\%-43\%) with $0.1 \%$ formic acid on a Waters nanoACQUITY UPLC system (Waters Corporation) and analyzed on a coupled Thermo Scientific Orbitrap Fusion Tribrid mass spectrometer (Thermo Scientific) as described (Williamson et al., 2016). Full scans were acquired at a resolution of 120,000 , and precursors were selected for fragmentation by higher-energy collisional dissociation (normalized collision energy at $30 \%$ ) for a maximum 3 -second cycle. Tandem mass spectra were searched against a UniProt human reference proteome using a SEQUEST HT algorithm (Eng et al., 2008) with a maximum precursor mass error tolerance of $10 \mathrm{ppm}$. Resulting hits were validated at a maximum false discovery rate of 0.01 using a semisupervised machine learning algorithm, Percolator (Käll et al., 2007). Abundance ratios were measured by comparing the mass spectrometer 1 peak volumes of peptide ions, whose identities were confirmed by mass spectrometer 2 sequencing as described above. Label-free quantifications were performed using an aligned Accurate Mass and Retention Time cluster quantification algorithm (Qi et al., 2012). Pathway and gene ontology analysis were performed with Qiagen Ingenuity and Panther Gene ontology databases, as described (Krämer et al., 2014; Mi et al., 2017). Proteins showing at least a doubling in expression as compared with untreated cells, with an FDR-adjusted ANOVA $P$ value of $<0.05$, were considered significantly changed and used for further analysis. FDR-corrected Fisher's exact $P$ values $<0.05$ using a previously described procedure for multiple testing were used in the gene ontology analyses to identify biologic processes, molecular functions, and cellular components associated with observed protein changes (Benjamini and Hochberg, 1995). The Ingenuity pathway data base was used to predict canonical pathways and upstream regulators according to the proteins that were significantly different using an absolute activation z-score $>2$ for at least one condition with a Fisher's exact test $P$ value $<0.05$.

Lactate Dehydrogenase/Cytotoxicity Assay. Cells were seeded 5000 cells per well in 96-well plates, cultured overnight, and treated for 48 hours with the indicated dose of compound SF-3-030. Cytotoxicity via LDH release was measured according to the manufacturer's instructions using the CyQUANT LDH Cytotoxicity Assay Kit (Thermo Fisher Scientific).

Statistical Analysis. Graphical statistical analysis was performed using a one-tailed ANOVA with a 95\% confidence interval using GraphPad-Prism version 5.01. Three biologic replicates were chosen for data subjected to statistical analysis. The data represent descriptive statistics and were used to summarize key findings. Results were considered statistically significant if the $P$ value is less than 0.05 .

\section{Results}

The Double Bond in the Sulfur Heterocycle Is Required for Biologic Activity. Our previous studies described a novel class of small molecules containing a 1,1dioxido-2,5-dihydrothiophen-3-yl 4-benzenesulfonate scaffold that selectively inhibited BRAF mutated melanoma cells containing constitutively active ERK1/2 signaling (Samadani et al., 2015). A limited structure-activity relationship study was performed to determine key chemical features required for the compound's biologic activity. Modifications of the parent compound, SF-3-026, focused on the benzene ring, the linker region connecting the two ring structures, and removal of the double bond in the sulfur heterocycle (Fig. 1). The analogs were tested at a single high dose for effects on viability of cancer cell lines containing BRAF mutations (A375, RPMI-7951, and
SK-Mel-28), NRas mutation (HL60), or no known mutations in ERK1/2 pathway proteins (HeLa and Jurkat) (Fig. 1). RPMI7951 cells are inherently resistant to BRAF targeted inhibitors because of upregulated expression of MAP3K8 (Johannessen et al., 2010). In agreement with our previous studies (Samadani et al., 2015), SF-3-026, SF-3-027, SF-3-029, and SF-3-030 showed selective inhibition of cancer cell lines with activating mutations in the ERK1/2 pathway (Fig. 1). The inhibitory effects were lost in compounds in which the double bond in the sulfur heterocycle was removed (Fig. 1). Select modifications to the benzene group or linker in the absence of the double bond in the sulfur heterocycle had no effect on cell viability, indicating the 1,1-dioxido-2,5-dihydrothiophen-3-yl moiety was essential for the compound's biologic activity (Fig. 1).

Previous studies showed that catalytic site inhibitors of MEK1/2 and ERK1/2 are selective for cells with activating BRAF or Ras mutations (Yeh et al., 2007; Morris et al., 2013). Similarly, we determined the $\mathrm{IC}_{50}$ values for $\mathbf{S F - 3 - 0 3 0}$ and ATP-dependent/catalytic site inhibitors of MEK1/2 and ERK1/2 in four cancer cell lines and showed that all compounds favored inhibition of cells containing activating BRAF and Ras mutations (Supplemental Table 1). The reported doubling times for A375, HeLa, and Jurkat cells are 25-30 hours, compared with $\sim 50$ hours for the RPMI-7951 cells, indicating that each compound's potency is independent of cell growth rates. These findings provide additional support that SF-3-030 inhibitory effects on cell growth are correlated with ERK1/2 activity.

The effects of SF-2-110 (inactive control), SF-3-026, and SF-3-030 (Supplemental Fig. 1A) on cell viability were further evaluated in dose-response assays using four melanoma cell lines containing either BRAF or NRas mutations. As expected, SF-2-110 did not affect the viability of any of the cell lines up to $50 \mu \mathrm{M}$ (Supplemental Fig. 1, B-E). In contrast, SF-3-026 and SF-3-030 inhibited all cell lines with $\mathrm{IC}_{50}$ values in the 5-10 $\mu \mathrm{M}$ range, with SF-3-030 showing the highest potency against SK-MEL-28 and SK-MEL-2 cells (Supplemental Fig. $1, \mathrm{~B}-\mathrm{E})$. These findings indicate that the sensitivity of cancer cells in vitro to the lead compounds is independent of the BRAF or NRas mutational status.

SF-3-030 Directly Interacts with ERK2 through Noncovalent and Covalent Mechanisms. Given the higher inhibitory potency of SF-3-030, we evaluated whether this compound directly interacted with ERK2. Using STD-NMR, it was confirmed that SF-3-030 interactions with ERK2 occur primarily through a reversible interaction of the naphthalene group and the sulfur heterocycle (Supplemental Fig. 2). However, the requirement for the double bond in the sulfur heterocycle of SF-3-030 for biologic activity suggested that the compound's mechanism of action involves the formation of covalent adducts with cysteine residues. Two possible SF-3030 adducts could result from a Michael addition or a substitution reaction that would increase the molecular mass of a cysteine by 324 or $116 \mathrm{Da}$, respectively (Fig. 2A). To test this, ERK2 was incubated with SF-3-030, and covalent adducts were analyzed by high-resolution liquid chromatographytandem mass spectrometry. Of the seven cysteine residues in ERK2, cysteine $252(\mathrm{C} 252)$ was the predominant residue modified by a 116-Da mass, suggesting a substitution reaction and decomposition of SF-3-030 (Fig. 2; Supplemental Fig. 3). C252 is located near the DEF motif-recruitment site, which is 


\begin{tabular}{|c|c|c|c|c|c|c|c|c|c|}
\hline \multirow[t]{2}{*}{ Code Number } & \multirow[t]{2}{*}{ Structure } & \multirow[t]{2}{*}{$\mathbf{x}$} & \multirow{2}{*}{$\mathbf{R}$} & \multicolumn{6}{|c|}{ Cell Viability (\% of vehicle) } \\
\hline & & & & A375 & RPMI-7951 & SK-MEL-28 & HL-60 & HeLa & Jurkat \\
\hline SF-2-110 & & $\mathrm{O}$ & & $115 \pm 3$ & $98 \pm 7$ & $99 \pm 4$ & $103 \pm 36$ & $97 \pm 6$ & $104 \pm 2$ \\
\hline SF-3-006 & O & NMe & & $105 \pm 1$ & $98 \pm 6$ & $96 \pm 6$ & $98 \pm 13$ & $96 \pm 6$ & $97 \pm 4$ \\
\hline SF-3-007 & & $\mathrm{NMe}$ & & $102 \pm 2$ & $91 \pm 2$ & $93 \pm 5$ & $76 \pm 17$ & $92 \pm 1$ & $79 \pm 2$ \\
\hline SF-3-008 & & NMe & & $86 \pm 1$ & $100 \pm 4$ & $93 \pm 5$ & $64 \pm 31$ & $80 \pm 2$ & $75 \pm 9$ \\
\hline SF-3-009 & & NMe & & $98 \pm 4$ & $67 \pm 3$ & $90 \pm 6$ & $88 \pm 26$ & $85 \pm 4$ & $97 \pm 4$ \\
\hline SF-3-010 & & NMe & & $112 \pm 5$ & $117 \pm 9$ & $84 \pm 7$ & $99 \pm 33$ & $78 \pm 5$ & $79 \pm 3$ \\
\hline SF-3-011 & & $\mathrm{NH}$ & & $102 \pm 3$ & $110 \pm 1$ & $98 \pm 7$ & $131 \pm 49$ & $90 \pm 5$ & $97 \pm 2$ \\
\hline SF-3-013 & & NBn & & $106 \pm 1$ & $105 \pm 4$ & $94 \pm 3$ & $89 \pm 20$ & $86 \pm 6$ & $86 \pm 2$ \\
\hline SF-3-017 & & $\mathrm{NEt}$ & & $111 \pm 5$ & $105 \pm 6$ & $98 \pm 5$ & $97 \pm 23$ & $92 \pm 7$ & $94 \pm 2$ \\
\hline SF-3-018 & & NiPr & & $106 \pm 1$ & $102 \pm 9$ & $93 \pm 4$ & $82 \pm 20$ & $88 \pm 3$ & $89 \pm 2$ \\
\hline SF-3-026 & & $\mathrm{O}$ & & $5.2 \pm 1.0$ & $0.10 \pm 0.04$ & $36 \pm 13$ & $0.4 \pm 0.2$ & $49 \pm 7$ & $64 \pm 20$ \\
\hline SF-3-027 & & $\mathrm{O}$ & & $5.1 \pm 1.0$ & $0.10 \pm 0.04$ & $6.9 \pm 4.0$ & $0.6 \pm 0.2$ & $26 \pm 6$ & $19 \pm 7$ \\
\hline SF-3-029 & & $\mathrm{O}$ & & $8.2 \pm 1.0$ & $0.20 \pm 0.03$ & $9.4 \pm 5.0$ & $1.8 \pm 1.0$ & $26 \pm 4$ & $35 \pm 11$ \\
\hline SF-3-030 & & $\mathrm{O}$ & & $4.9 \pm 1.0$ & $0.10 \pm 0.03$ & $26 \pm 7$ & $1.3 \pm 0.3$ & $52 \pm 7$ & $70 \pm 12$ \\
\hline
\end{tabular}

Fig. 1. Effects of SF-3-026 and analogs on proliferation of cancer cell lines. Data show percent proliferation compared with controls (100\%) for A375, RPMI-7951, SK-Mel-28, HL-60, HeLa, or Jurkat cells after treatment with $100 \mu \mathrm{M}$ of each test compound for 48 hours. The mean and S.D. from three independent experiments are shown. 
A

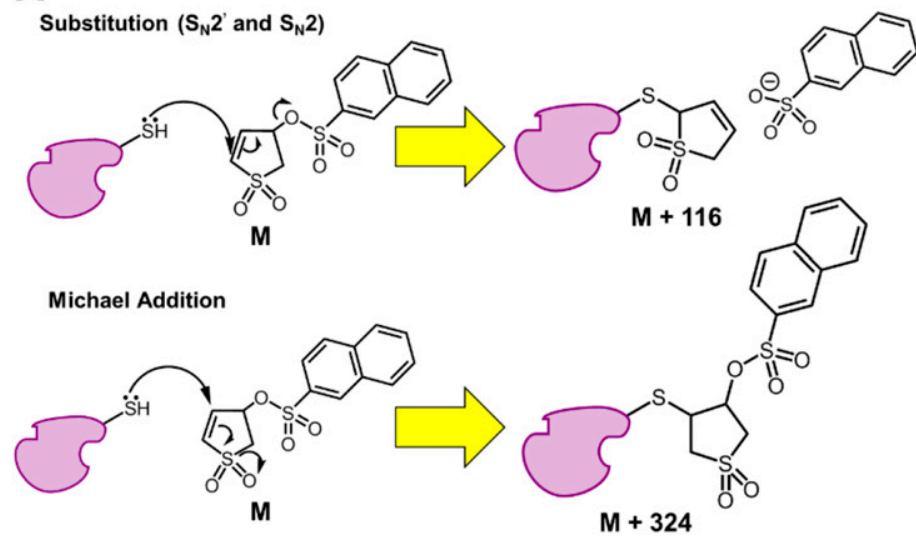

B

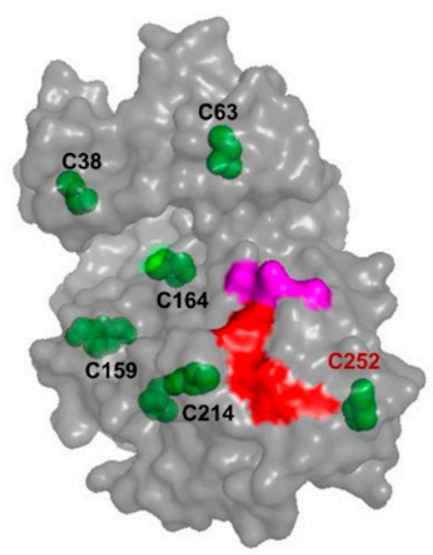

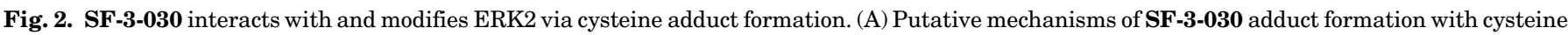

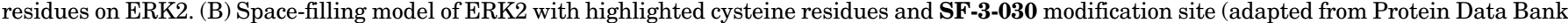

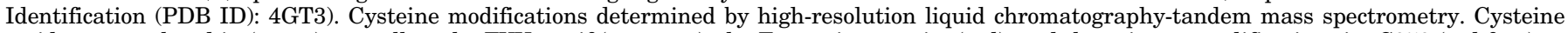
residues are colored in (green), as well as the TXY motif (magenta), the F-recruitment site (red), and the primary modification site C252 (red font).

consistent with its proposed mechanism of targeting DEF motif-containing substrates (Samadani et al., 2015). Other major MAP kinases, such as p38 $\alpha$ and c-Jun N-terminal kinase 1 , do not have a cysteine located at this site. We have also previously determined that SF-3-030 had no effect on p38 MAPK signaling (Samadani et al., 2015), suggesting that SF-3-030 is not acting as a random alkylating agent or affecting similar MAP kinases. These data suggest that the initial binding interactions of SF-3-030 with ERK2 involve noncovalent interactions that position the compound to form a covalent bond between $\mathrm{C} 252$ and the sulfur heterocycle group.

SF-3-030 Selectively Regulates ERK-Dependent Immediate Early Gene Expression. We next examined relative protein levels of ERK1/2-regulated immediate early genes (IEGs). Previously, we showed that a 1-hour treatment with SF-3-030 caused differential changes in AP-1 protein levels characterized by inhibition of Fra-1, FosB, and the alternative splice variant FosB2 but no effect on c-Fos levels (Samadani et al., 2015). The relative levels of these proteins along with other AP-1 members were examined in A375 cells treated for 0-24 hours with SF-3-030 or the ERK1/2 catalytic site inhibitor SCH772984 as a positive control. Similar to our previous studies (Samadani et al., 2015), a 1 hour of treatment with SF-3-030 or SCH772984 had little effect on c-Fos levels (Fig. 3A). However, after 2 hours of treatment, c-Fos increased only with SF-3-030 and persisted for up to 24 hours (Fig. 3A). Another major component of the AP-1 complex, c-Jun, increased with either SF-3-030 or SCH772984 treatments (Fig. 3A). It should be noted that the increased levels of c-Fos and c-Jun do not correlate with increased AP-1 activity, which we have shown to be inhibited in these and other cell lines treated with SF-3-030 (Samadani et al., 2015; Defnet et al., 2019).

Other Fos family members, including FosB/B2 and Fra-1 decreased in cells treated with SCH772984 (Fig. 3A). Cells treated with SF-3-030 also showed decreased Fra-1 levels, whereas FosB/B2 levels transiently decreased, followed by an increase after 4 hours as observed with c-Fos (Fig. 3A). The levels of c-Myc, a potential target for treating melanoma (Polsky and Cordon-Cardo, 2003; Korkut et al., 2015), showed a rapid inhibition by SF-3-030 when compared with SCH772984 (Fig. 3A). Interestingly, c-Myc levels increased back to basal levels after 24 hours of treatment with SF-3-030 (Fig. 3A). However, this dose of SF-3-030 is lethal to $~ 90 \%$ of A375 cells after 48 hours of exposure (Supplemental Fig. 1B). A transient increase in the ERK1/2-mediated phosphorylation of Elk-1, a ternary complex factor involved in regulating $c$-fos and other IEGs (Shaw and Saxton, 2003), was observed in SF3-030-treated cells and correlated with ERK1/2 phosphorylation (Fig. 3B). However, there was no evidence that MEK1/2, the upstream activator of ERK1/2, was activated, indicating SF-3-030 was acting at the level of ERK1/2 (Supplemental Fig. 4).

The dose-dependent effect of the active compounds SF-3026 and SF-3-030 on IEG levels was examined after a 4-hour exposure. The inactive control, SF-2-110, had no effect on c-Fos, c-Jun, Fra-1, FosB/B2, or c-Myc levels (Fig. 3C). In contrast, SF-3-026 and SF-3-030 caused a dose-dependent decrease in Fra-1, FosB/B2, and c-Myc protein levels but an increase in c-Jun and c-Fos (Fig. 3C). The increased potency of SF-3-030 in affecting IEG protein levels compared with the parent compound SF-3-026 is consistent with our previous studies (Samadani et al., 2015). It is also noted that SF-3-030 at a dose near its $\mathrm{IC}_{50}(\sim 5 \mu \mathrm{M})$ for inhibition of cell proliferation was as potent at inhibiting Fra-1 and c-Myc protein levels as the $5 \mu \mathrm{M}$ doses of AZD6244 or SCH77294 (Fig. 3C). Quantitative analysis of c-Fos, Fra-1, and c-Myc protein levels after exposure with $25 \mu$ M SF-2-110 or SF-3-030 is shown in Fig. 3D. Together, these findings demonstrate that the lead compounds can differentially affect ERK1/2-regulated transcription factors.

It was next determined whether the changes in IEG expression observed after 1 to 2 hours with SF-3-030 (Fig. 3A) coincided with the exposure time needed to induce an apoptotic response. In these experiments, A375 cells were exposed to SF3-030 for various times, washed to remove excess compound, and then incubated with serum-supplemented growth medium for a total of 24 hours. Apoptosis was evident after 2 hours of exposure to SF-3-030, as measured by the appearance of cleaved PARP (Fig. 3E). This exposure time also coincided with the loss of histone H3 phosphorylation at Ser10, which is 
A

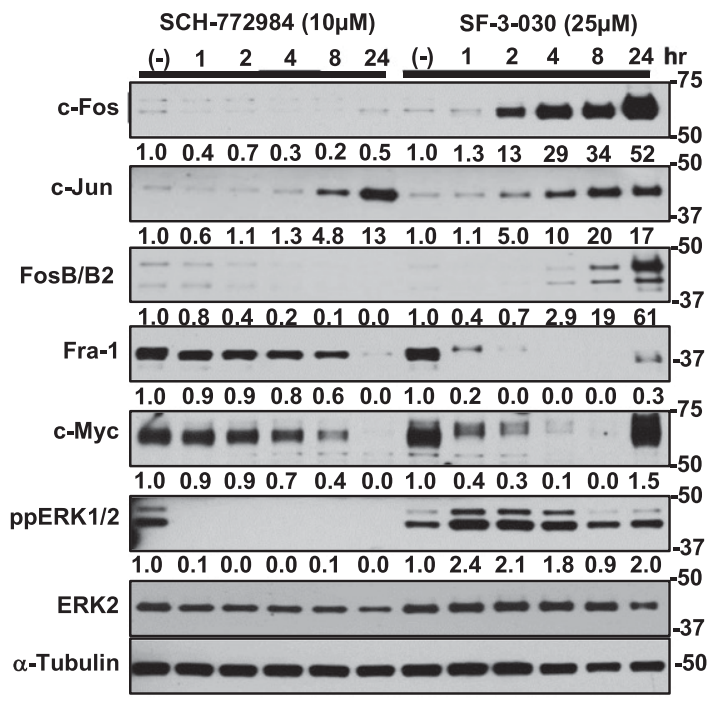

C

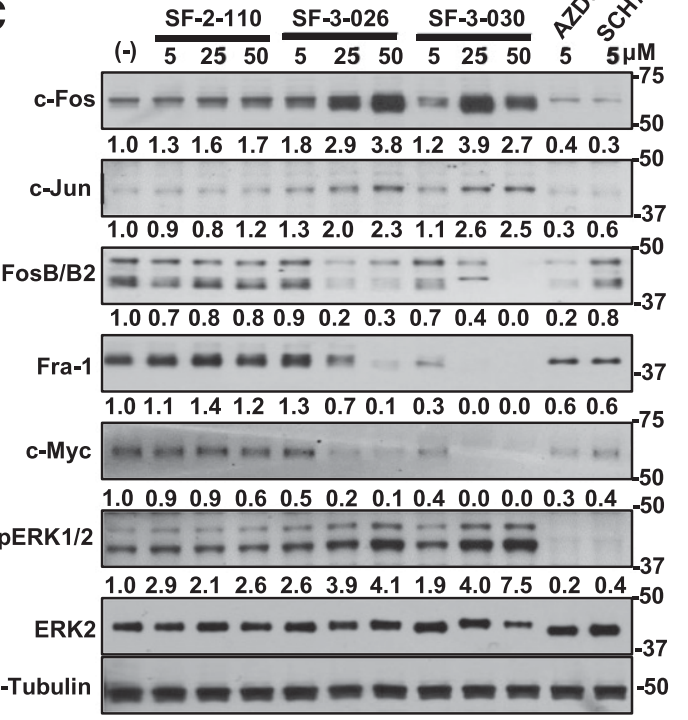

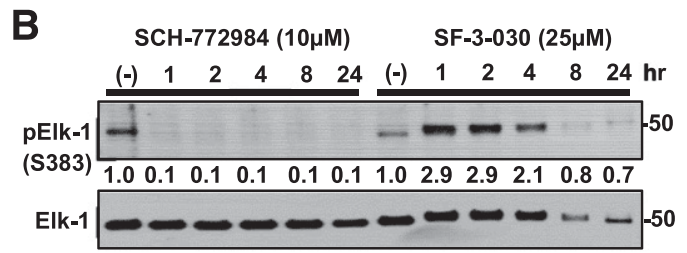

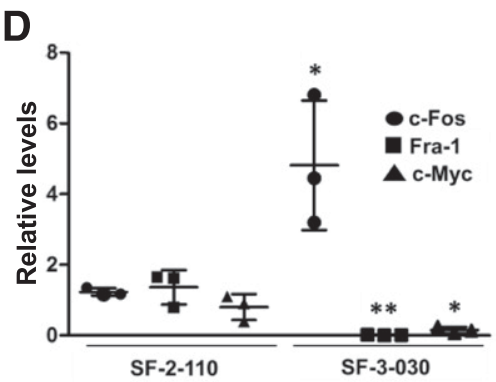

$\mathbf{E}$

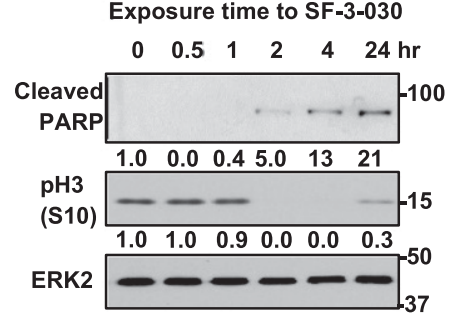

Fig. 3. Lead compounds differentially regulate IEG levels in A375 cells. (A) Immunoblots for c-Fos, c-Jun, FosB/B2, Fra-1, and c-Myc proteins in cells treated with $10 \mu \mathrm{M}$ SCH77294 or $25 \mu \mathrm{M}$ SF-3-030 for 0-24 hours. (B) Phosphorylated (S383) and total Elk-1 levels in cells treated with $10 \mu \mathrm{M}$ SCH77294 or $25 \mu \mathrm{M}$ SF-3-030 for 0-24 hours. (C) Cells treated for 4 hours with varying doses of SF-2-110, SF-3-026, or SF-3-030. Controls include untreated or cells treated with $5 \mu \mathrm{M}$ AZD6244 or SCH772984. Immunoblots show relative c-Fos, c-Jun, FosB/B2, Fra-1, and c-Myc protein levels. Levels of active ERK1/2 (ppERK1/2), total ERK2, and $\alpha$-tubulin are shown to demonstrate ERK1/2 pathway activity and equal protein loading in (A) and (C). (D) Relative quantification of c-Fos, Fra-1, and c-Myc protein levels after 4 hours of exposure with $25 \mu \mathrm{M}$ of SF-2-110 or SF-3-030. Mean and S.D. are from three independent experiments, and graph was determined via densitometry as described in the Materials and Methods. * and ** represent $P<0.05$ and $P<0.01$, respectively. (E) A375 cells were exposed to SF-3-030 for the indicated times and then incubated with fresh media without compound for a total incubation time of 24 hours. Lysates were immunoblotted for cleaved PARP, phosphorylated histone H3 (pH3), and total ERK2 for a loading control. Data are representative of three independent experiments. The numbers in each immunoblot represent the relative levels of protein, normalized to $\alpha$-tubulin, as determined by densitometry. Molecular mass markers $(\mathrm{kDa})$ are indicated on the right of each immunoblot.

a marker of mitosis and is observed during the transcription of selective IEGs in response to ERK1/2 pathway activation (Sassone-Corsi et al., 1999). Loss of histone H3 phosphorylation after 2 hours of exposure to SF-3-030 suggests that mitotic progression is inhibited and that phosphorylation of S10 is dispensable for regulating the transcription of IEG, such as c-Fos and c-Jun, as previously reported (Drobic et al., 2010).

SF-3-030 Regulates ERK1/2-Dependent and Independent Transcription. RNA sequencing analysis was used to measure early changes in gene expression patterns and identify on- and off-target effects of SF-3-030. Total RNA was isolated from A375 cells treated for 1 hour with an $\mathrm{IC}_{90}$ dose $(25 \mu \mathrm{M})$ of SF-3-030 or the MEK inhibitor AZD6244 $(10 \mu \mathrm{M})$ as a positive control for ERK1/2 pathway inhibition. The IC $_{90}$ dose for SF-3-030 was used, as it was determined to have no general cytotoxicity in LDH assays [data not shown;
(Defnet et al., 2019)]. Approximately 16,000 transcripts were identified in each condition, and statistically significant changes ( $\geq 1.5$-fold) in transcript expression between treated and untreated cells were reported. We identified 38 or 68 transcripts in cells treated with SF-3-030 or AZD6244, respectively, which showed significant decreases with 12 genes common to both treatments (Supplemental Table 2). ERK1/2 pathway targets involved in cell growth and survival that decreased with both treatments included $c-M y c, C T G F$, SOX11, and GATA3. AZD6244 also inhibited transcription of $c$-Fos, early growth reponse-1 (Egr-1), and IER3 genes, which are elevated in BRAF V600E mutated melanoma cells and may promote ERK-mediated growth of these tumor types (Pratilas et al., 2009). The inhibition of c-Myc by SF-3-030, AZD6244, and SCH772984 at the RNA and protein levels (Fig. 3) supports a shared mechanism of targeting ERKmediated signaling by these compounds. 
The data also revealed that AZD6244 inhibited many negative regulators of the ERK1/2 pathway, including Sprouty $(S P R Y)$ and dual specificity phosphatase transcripts (Supplemental Table 2). SPRY1 was the only negative regulator of ERK1/2 signaling that was inhibited by both AZD6244 and SF-3-030 (Supplemental Table 2). Additional growth-related genes that decreased only with AZD6244 treatment included regulators of epidermal growth factor receptor signaling including $c-F o s$, TGFA, and HBEGF (Supplemental Table 2). In contrast, only SF3-030 inhibited a unique set of growth- and angiogenesispromoting genes, including CYR61 (also called IGF-binding protein 10), which may promote tumor progression during hypoxia (Kunz et al., 2003), and BCL6, which has been associated with poor overall survival of patients with melanoma (Alonso et al., 2004).

There were 14 or 24 transcripts that increased $>1.5$-fold after treatment with SF-3-030 or AZD6244, respectively, relative to untreated cells, and only one gene, $C H M P 4 C$, was common to both treatments (Supplemental Table 2). CHMP4C is involved in sorting and delivery of endosomal vesicles to the lysosome and in cell cycle checkpoint control during cytokinesis (Carlton et al., 2012). Ingenuity pathway analysis (IPA) indicated that several genes upregulated in response to SF-3-030 treatment, including HMOX1, c-Fos, c-Jun, and DnaJ homolog subfamily B member 1 (Supplemental Table 2), were consistent with activation of an NRF2-mediated oxidative stress response. However, given that the exposure to SF-3-030 was only 1 hour, it is likely that other transcription factors besides NRF2 are regulating these genes at this time point.

Other genes upregulated by SF-3-030 included MAP3K14 (also called NF- $\kappa \mathrm{B}$-inducing kinase), which activates NF- $\kappa \mathrm{B}$, and the Egr-1 transcription factor, which may have tumor suppressor functions through regulation of p53 (Shin et al., 2006). Transcripts that increased only in response to AZD6244 treatment included SOX2 and FOXD3, which are associated with survival of melanoma-initiating cells, metastasis, and drug resistance (Abel et al., 2013; Santini et al., 2014). The activation status of the top 50 upstream regulators predicted to be affected after treatment with SF-3-030 or AZD6244 was determined using IPA software. Using Z-scores $>2$ or $<-2$ to indicate activation or inactivation, respectively, and $P$ values of $<0.03$, AZD6244 predictably inhibited many regulators of ERK1/2 and other MAP kinase signaling pathways (Supplemental Table 3). In contrast, SF-3-030 was predicted to affect only six regulators, including activation of $E g r-1$, as indicated previously (Supplemental Table 3). Given some overlap with AZD6244-regulated genes, these data suggest that SF-3-030 treatment affects fewer cellular signaling events than AZD6244 and that early MAP kinase signaling events largely remain intact.

SF-3-030 Enhances Markers of Mitochondrial Dysfunction and Oxidative Stress. To gain further insight into the mechanism of SF-3-030 inhibition of cell proliferation, we next used high-resolution liquid chromatographytandem mass spectrometry to determine the changes in levels of soluble proteins in A375 cells treated for 4 or 12 hours with SCH772984 or SF-3-030. After 4 hours, 22 (SF-3-030) or 16 (SCH772984) proteins were identified that showed significant $(P<0.05)$ changes compared with untreated cells (Supplemental Table 4). A summary of proteins that showed statistically significant increases or decreases ( $\geq 1.5$-fold) with SF-3-030 or SCH772984 as compared with untreated controls is shown in Table 1. At the 4-hour time point, only one protein, adenosylhomocysteinase (AHCY), which regulates the generation of the $S$-adenosylmethionine methyl donor, was inhibited with SF-3-030 or SCH772984 treatment. Proteins that increased after exposure to SF-3-030 included the zinc finger protein 774 (ZNF774), which has recently been shown to have tumor suppressor functions in hepatocellular carcinoma (Guan et al., 2020); transcription factor 4 (TCF4), which regulates cell differentiation; and HMOX1 (Table 1). SF-3030 decreased cytochrome c-type heme lyase (HCCS) levels, suggesting defects in mitochondrial electron transport function (Table 1). SCH772984 increased levels of the transmembrane and TPR repeat-containing protein 1, which indicated disruption of calcium homeostasis in the endoplasmic reticulum (Sunryd et al., 2014), and decreased the tetratricopeptide repeat protein, whose function is currently unknown (Table 1).

After 12 hours, 44 and 48 proteins showed significant changes $(P<0.05)$ with SF-3-030 and SCH772984 treatment, respectively, as compared with untreated cells (Supplemental Table 5). Proteins that increased or decreased $\geq 1.5$-fold with SF-3-030 or SCH772984 treatments relative to untreated controls are shown in Table 2. Common to SF-3-030 or SCH772984 treatments was increased levels of TCF4 and synthesis of cytochrome c oxidase 2 (SCO2) and decreased levels of a protein involved in ribosome biogenesis (RSL24D1). $\mathrm{SCO} 2$ has been associated with p53-mediated apoptosis signaling pathways through the generation of ROS (Madan et al., 2013).

Unique to SF-3-030 was a significant increase in the OSGIN1 protein (Table 2), which is regulated by oxidized lipids and NRF2 during oxidative stress to promote cytochrome c release from mitochondria (Li et al., 2007). Mediator

\section{TABLE 1}

High-resolution liquid chromatography-tandem mass spectrometry analysis of proteins that increase or decrease $\geq 1.5$-fold after treatment with SF-3-030 or SCH772984 for $4 \mathrm{~h}$ relative to untreated A375 cells (set at 1)

Protein changes common to both SF-3-030 and SCH772984 are italicized.

\begin{tabular}{cc}
\hline Proteins that increase with SF-3-030 & Fold increase \\
\hline ZNF774 & 16.28 \\
MEMO1 & 10.55 \\
TTC39C & 5.18 \\
MYO18A & 4.18 \\
TCF4 & 2.24 \\
HMOX1 & 1.77 \\
Proteins that decrease with SF-3-030 & Fold decrease \\
HCCS & 3.35 \\
KIAA1211 & 2.24 \\
ACTN2 & 1.66 \\
AHCY & 1.53 \\
\hline Proteins that increase with SCH772984 & Fold increase \\
\hline TMTC1 & 5.15 \\
SRGAP1 & 1.47 \\
Proteins that decrease with SCH772984 & Fold decrease \\
TTC29 & 2.44 \\
AHCY & 1.48 \\
\hline
\end{tabular}

ACTN2, $\alpha$-actinin-2; KIAA1211, Capping protein inhibiting regulator of actin dynamics; MYO18A, unconventional myosin-XVIIIa; SRGAP1, SLIT-ROBO Rho GTPase-activating protein 1; (TMTC1), transmembrane and TPR repeat-containing protein 1; TTC29, tetratricopeptide repeat protein 29; TTC39C, Tetratricopeptide repeat protein $39 \mathrm{C}$ 


\section{TABLE 2}

High-resolution liquid chromatography-tandem mass spectrometry analysis of proteins that increase or decrease $\geq 1.5$-fold after treatment with SF-3-030 or SCH772984 for $12 \mathrm{~h}$ relative to untreated A375 cells (set at 1)

Protein changes common to both SF-3-030 and SCH772984 are italicized.

\begin{tabular}{lc}
\hline A. & \\
Proteins that increase with SF-3-030 & Fold increase \\
\hline OSGIN1 & 21.04 \\
ZNF774 & 18.18 \\
TCF4 & 10.95 \\
PXDN & 9.43 \\
Protein MEMO1 & 8.37 \\
TTC39C & 7.31 \\
MYO18A & 4.50 \\
SCO2 & 3.89 \\
Jouberin (AHI1) & 3.12 \\
HMOX1 & 3.02 \\
Protein moonraker (KIAA0753) & 2.26 \\
ASB6 & 2.07 \\
REPS1 & 1.98 \\
NOC2L & 1.73 \\
Sickle tail protein homolog (KIAA1217) & 1.71 \\
NCAPD3 & 1.49 \\
Proteins that decrease with SF-3-030 & Fold decrease \\
Myomegalin (PDE4DIP) & 2.80 \\
HCCS & 2.49 \\
AP1M1 & 2.24 \\
CNGB1 & 2.11 \\
FOXR2 & 1.76 \\
GRIP2 & 1.72 \\
SUN1 & 1.68 \\
RSL24D1 & 1.66 \\
KIAA1211 & 1.62 \\
INTS2 & 1.51 \\
\hline
\end{tabular}

\section{B.}

Proteins that increase with SCH772984

FOXD3
PKP2
PABPC3
WDR59
SCO2
TCF4
KIAA1211
HBS1L
PDIA3
GFPT2
roteins that decrease with SCH772984
CACNB4
PDE5A
RFC4
RNMT
ATP11A
TFAP2A
RSL24D1
DCN
HK2
ABHD5
COL1A1

ABHD5, Abhydrolase domain-containing protein 5; AHI1, Abelson helper integration site 1 protein homolog; AP1M1, AP-1 complex subunit $\mu$-1; ASB6, ankyrin repeat and SOCS box protein 6; ATP11A, ATPase class VI type 11A; CACNB4, Calcium channel voltage-dependent subunit $\beta$ 4; CNGB1, cyclic nucleotide-gated cation channel $\beta$-1. COL1A1, collagen $\alpha$-1(I) chain; DCN, decorin; FOXR2, forkhead box protein R2; GFPT2, glutamine-fructose-6-phosphate aminotransferase 2; GRIP2, glutamate receptor-interacting protein 2; HBS1L, HBS1-like protein; HK2, hexokinase-2; INTS2, integrator complex subunit 2; KIAA1211, uncharacterized protein KIAA1211; MYO18A, unconventional myosin-XVIIIa; NCAPD3, condensin-2 complex subunit D3; NOC2L, nucleolar complex protein 2 homolog; PABPC3, polyadenylate-binding protein 3; PDE5A, phosphodiesterase 5A cGMP-specific; PDIA3, protein disulfide-isomerase A3; PKP2, plakophilin-2; PXDN, peroxidasin homolog; REPS1, RalBP1-associated Eps domain-containing protein 1; RFC4, replication factor C subunit 4; RNMT, mRNA cap guanine-N7 methyltransferase; SUN1, Sad1/unc-84 protein-like 1; TFAP2A, transcription factor AP-2- $\alpha$; TTC39C, chromosome 18 open reading frame 17_isoform CRA_c; WDR59, WD repeatcontaining protein 59. KIAA0753, moonraker; KIAA1217, Sickle tail protein homolog; PDE4DIP, Phosphodiesterase 4D-interacting protein. of ErbB2-driven cell motility 1 (MEMO1), a protein involved in the sustained production of ROS (MacDonald et al., 2014), was also increased in cells exposed to SF-3-030 (Table 1; Table 2). As was observed after 4 hours, HCCS levels were also decreased after a 12-hour exposure to SF-3-030 (Table 1; Table 2), further supporting compromised mitochondria function. Together, these findings indicate $\mathbf{S F - 3 - 0 3 0}$ induced signaling events that reduce mitochondrial function and increase ROS. After 12 hours of exposure to SCH772984, a significant induction of FOXD3 was observed (Table 2), which is consistent with previous studies that show upregulation of this protein promotes resistance to Raf and MEK inhibitors (Abel et al., 2013). Immunoblot analysis confirmed the induction of FOXD3 expression in A375 cells treated with SCH772984 but not in SF-3-030-treated cells (Supplemental Fig. 5).

Based on the observed changes in protein levels, IPA pathway analysis suggested a potential role for SF-3-030 induction of NRF2-mediated oxidative stress response and heme degradation in agreement with mitochondrial dysfunction. These findings are consistent with previous studies that implicate the ERK1/2 pathway in regulating oxidative phosphorylation in melanoma cells and the induction of ROS after treatment with inhibitors of BRAF and MEK1/2 (Haq et al., 2013; Cesi et al., 2017; Yuan et al., 2020). Immunoblot analysis confirmed that SF-3-030 treatment rapidly induced NRF2 levels that were sustained for at least 24 hours and that NRF2 induction could be inhibited by cotreatment with the ROS inhibitor NAC (Fig. 4).

SF-3-030 Inhibition of A375 Cell Proliferation Is Dependent on ROS Induction but Independent of NRF2. The transcriptome and proteome data provided evidence for the generation of ROS in cells treated with SF-3-030. We confirmed the increased ROS production in A375 cells after a 1-hour treatment with SF-3-030, which was partially

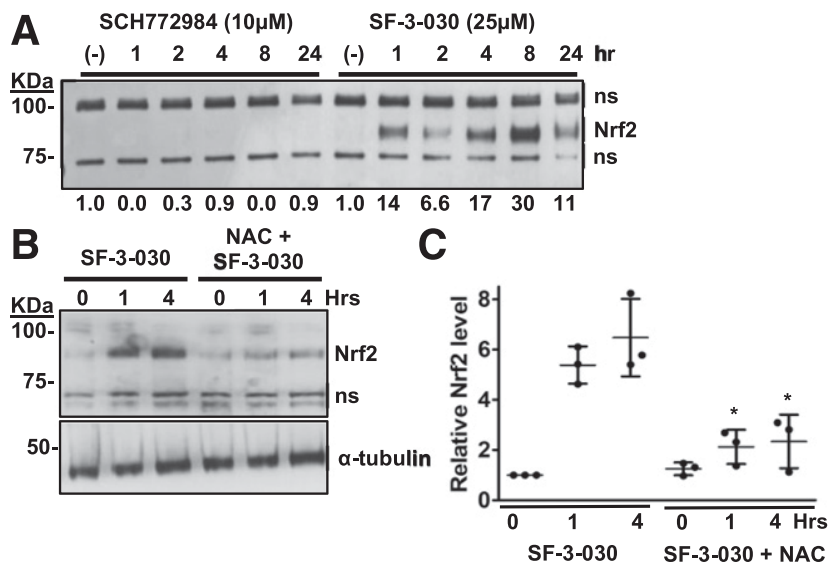

Fig. 4. SF-3-030 induces NRF2 levels. (A) Immunoblot analysis of NRF2 in A375 cells treated with $10 \mu \mathrm{M}$ SCH772984 or $25 \mu \mathrm{M}$ SF-3-030 for $0-24$ hours. (B) NRF2 levels in A375 cells treated with $25 \mu \mathrm{M} \mathrm{SF-3-030} \pm 5 \mathrm{mM}$ NAC for 0,1 , or 4 hours. Nonspecific bands that crossreact with the NRF2 primary antibody are indicated (ns) for (A) and (B). The graph in (C) shows the densitometry quantitation of NRF2 under the conditions described in (B). Mean and S.D. are from three independent experiments. * indicates statistical significance compared with SF-3-030 treatment only $(P<0.05)$. The numbers in each immunoblot represent the relative levels of protein, normalized to $\alpha$-tubulin, as determined by densitometry. Molecular mass markers are indicated on the left of each immunoblot. 
inhibited by cotreatment with several ROS inhibitors (Fig. 5A). NAC was the only ROS inhibitor that restored A375 cell proliferation in the presence of SF-3-030 (Fig. 5B). Similarly, only NAC reversed SF-3-030 induction of c-Fos (Fig. 5C). Although these data indicate that NAC may mitigate ROS production by SF-3-030 to restore A375 cell proliferation, there was also the possibility that NAC directly forms adducts with SF-3-030, which would reduce the compound's effective concentration and biologic activity. However, using thin-layer chromatography to separate compounds, we found no evidence that NAC directly interacted with SF-3-030 after a 1- or 24hour incubation in both PBS and methanol solvent systems (data not shown).

Induction of NRF2 has been implicated in protecting against oxidative stress induced by anticancer drugs and may contribute to drug resistance (Telkoparan-Akillilar et al., 2019). To assess whether NRF2 induction protected cells from SF-3-030 inhibition, we cotreated A375 cells with the NRF2 inhibitors ML-385 or brusatol (Ren et al., 2011; Olayanju et al., 2015; Singh et al., 2016). SF-3-030 and sulforaphane, as a positive control, induced NRF2 and target genes such as HMOX1, OSGIN1, NAD(P)H dehydrogenase [quinone] 1 (NQO1), GCLM, and SRXN1 (Fig. 6, A and B; Supplemental Fig. 6A). In contrast, protein levels for other NRF2-regulated genes, including aldo-keto reductase family 1 member B10, GCLC, glucose-6-phosphate dehydrogenase, cystine/glutamate transporter/solute carrier family 7 member 11, and thioredoxin reductase 1 were not induced by $\mathbf{S F - 3 - 0 3 0}$ (Supplemental Fig. 7).

As compared with sulforaphane, SF-3-030 induced a more robust and sustained induction of $\mathrm{HMOX} 1$, indicating qualitative differences in NRF2 activators. Brusatol was a potent inhibitor of SF-3-030 or sulforaphane induction of NRF2 and its target genes. Although ML-385 inhibited HMOX1 induced by sulforaphane after 24 hours (Supplemental Fig. 6, A and B), it was not as effective as brusatol at inhibiting NRF2 signaling induced by SF-3-030 (Fig. 6A). ML-385 had no effect on cell proliferation up to $50 \mu \mathrm{M}$; however, brusatol inhibited A375 cells in a dose-dependent manner (Fig. 6, C and D). Cotreatment with brusatol or ML-385 had little effect on SF-3-030 or sulforaphane inhibition of A375 cell proliferation, indicating NRF2 induction was dispensable for the cell response to these compounds (Fig. 6, E and F; Supplemental Fig. 6, C and D). Dose-dependent inhibition of A375 cells by sulforaphane or brusatol alone supports the high sensitivity these cells have to fluctuations in ROS (Meierjohann, 2014). Together, these data indicate that SF-3-030-mediated inhibition of A375 melanoma cell proliferation is through a mechanism that is ROSdependent and NRF2-independent.

\section{Discussion}

The first objective of the current studies was to elucidate the structure-activity relationship of a 1,1-dioxido-2,5-dihydrothiophen-3-yl 4-benzenesulfonate chemical scaffold that we previously identified to selectively inhibit cancer cells containing activated ERK1/2 signaling (Samadani et al., 2015). The dependence on a double bond in the sulfur heterocycle for biologic activity indicated the compound's mechanism of action involved the formation of covalent adducts with cysteine residues on ERK1/2 and perhaps other proteins through Michael addition chemistry. SF-3-030 was confirmed via STD-NMR and mass spectrometry-based analyses to interact with ERK2 via noncovalent interactions and the formation of a covalent adduct on C252 near the FRS. Although the formation of covalent adducts raises the concern

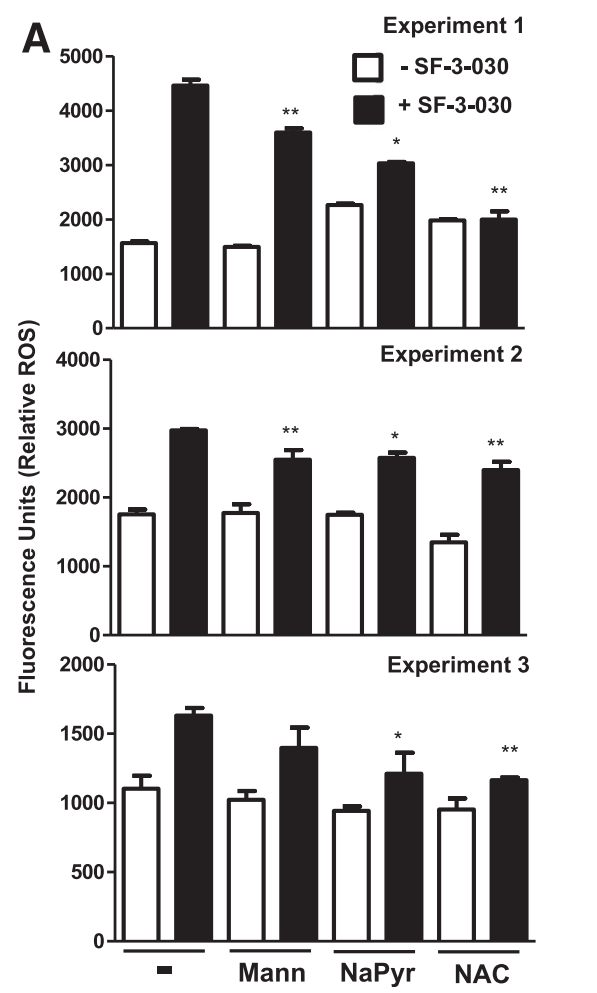

B
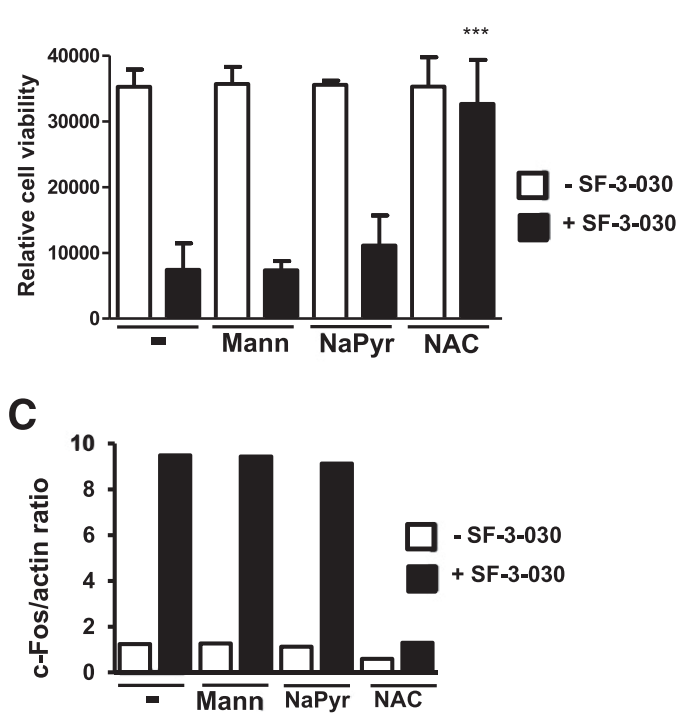

Fig. 5. SF-3-030 induction of ROS mediates inhibition of A375 cell proliferation. (A) ROS was measured by CellROX Deep Red reagent fluorescence in A375 cells treated with $25 \mu \mathrm{M}$ SF-3-030 in the absence (white bars) or presence (black bars) of the following ROS inhibitors (ROSi): $10 \mathrm{mM}$ sodium pyruvate (NaPyr), $100 \mathrm{mM}$ mannitol (Mann), or $10 \mathrm{mM}$ NAC for 60 minutes. Graphs represent three independent experiments, and each data point represents the mean \pm S.D. from three wells with four fields of view per well and each field containing between 500 and 1000 cells. Statistical significance was determined within each experiment (* and ** represent $P<0.05$ and $P<0.01$, respectively). (B) A375 cell viability was measured after 48 hours in untreated or SF-3-030-treated cells in the absence (white bars) or presence (black bars) of the ROS inhibitors at the concentrations described in (A). The mean and S.D. for cell proliferation are from three independent experiments. ** indicates statistical significance $(P<0.01)$ compared with SF3-030 treatment only. (C) Relative levels of c-Fos after 4 hours of treatment with SF-3-030 alone (white bars) or in combination with the indicated ROS inhibitors (black bars) at the concentrations indicated in (A). Relative c-Fos levels were determined by ProteinSimple immunoanalysis and normalized to total $\beta$-actin. 

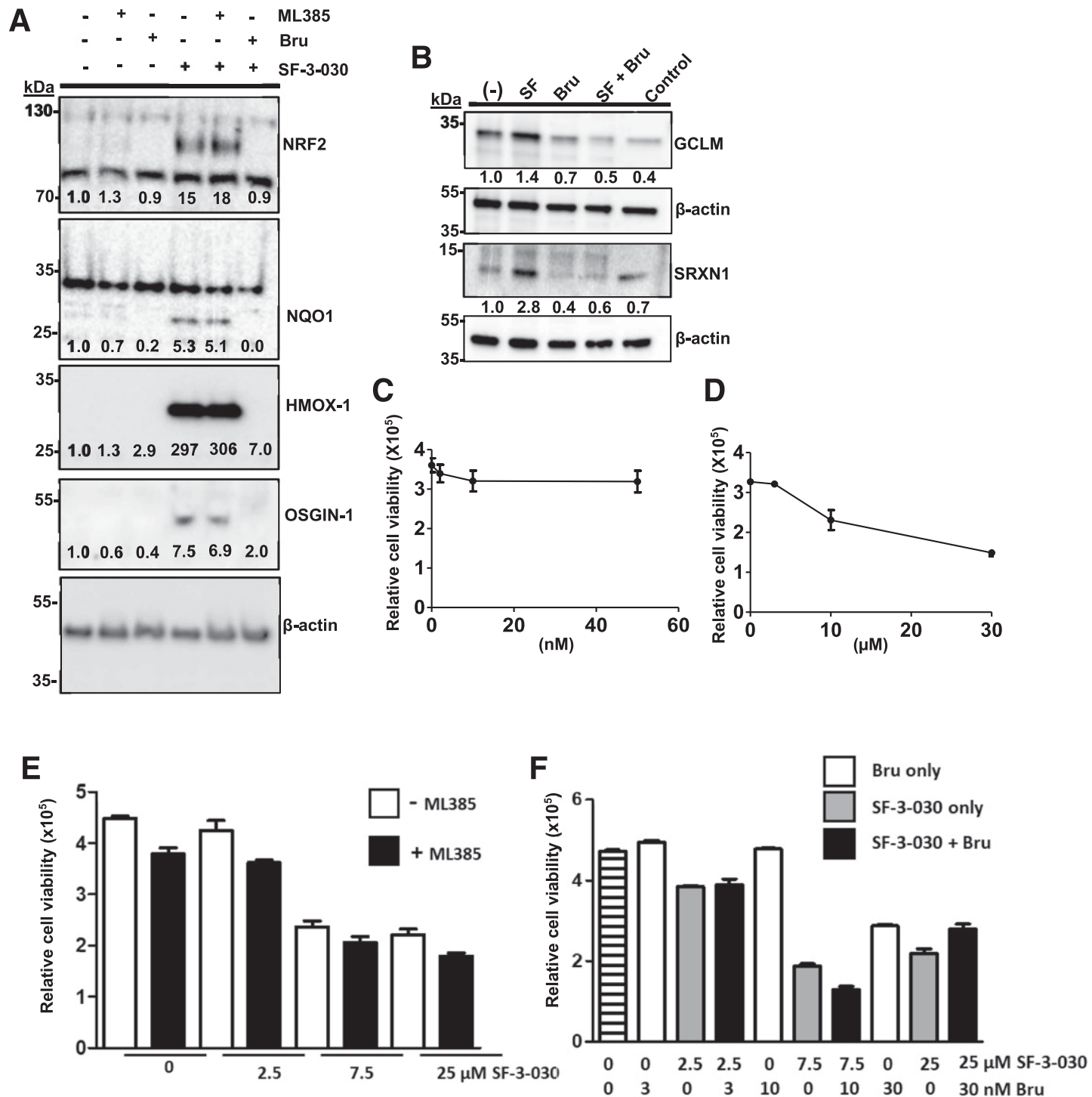

Fig. 6. NRF2 inhibitors do not affect SF-3-030 inhibition of A375 cell proliferation. (A) A375 cells were treated for 8 hours with $25 \mu \mathrm{M}$ SF-3-030 in the absence or presence of ML-385 (50 $\mu \mathrm{M}$ ) or brusatol (Bru; $30 \mathrm{nM}$ ). Lysates were immunoblotted for relative levels of NRF2, NQO1, HMOX1, and OSGIN1, as shown. (B) A375 lysates from cells treated for 8 hours with $25 \mu \mathrm{M}$ SF-3-030 (SF) in the absence or presence of Bru (30 nM) were immunoblotted for GCLM and SRXN1. Positive control lysates from HeLa and A549 cells were used for GCLM and SRXN1, respectively. The numbers in each immunoblot represent the relative levels of protein, normalized to $\beta$-actin, as determined by densitometry. Molecular mass markers are indicated on the left of each immunoblot. (C) A375 cell viability with varying doses of ML-385 or (D) brusatol. (E) Cell viability with varying doses of SF-3-030 in the absence (white bars) or presence (black bars) of $50 \mu \mathrm{M}$ ML-385. (F) Combination index with varying doses of SF-3-030 and brusatol. Untreated control cells are indicated with a striped column. Relative cell viability was measured after 48 hours, and data are representative of three independent experiments.

about specificity and off-target effects, we have found no evidence that SF-3-030 leads to changes in other MAP kinase signaling pathways or randomly interacts with cysteine using the ROS inhibitor NAC (data not shown). This indicates that the chemical structure of SF-3-030 confers target selectivity and prevents random interactions with sulfhydryl groups.

There is the potential that SF-3-030 forms a covalent adduct with Kelch-like ECH-associated protein 1 (KEAP1), the negative regulator of NRF2. KEAP1 has up to 21 cysteine residues that may be modified by chemical stressors to relieve KEAP1 inhibition and allow NRF2 activation (Dayalan Naidu and Dinkova-Kostova, 2020). Sulforaphane, which modifies KEAP1 on C151 to activate NRF2, showed qualitative differences in the expression of NRF2-regulated genes as compared with SF-3-030 (Fig. 6; Supplemental Fig. 6). It will be interesting to evaluate whether SF-3-030 also modifies KEAP1 cysteine residues and how they impact NRF2 functions. As indicated in the current studies, SF-3-030 induction of NRF2 did not appear to affect A375 cell proliferative responses to SF-3-030.

Despite past concerns about safety and off-target toxicity, advances in structure-function relationships and medicinal chemistry have generated renewed interest in the development of irreversible binding drugs for treating disease (Singh et al., 2011). There are several small-molecule anticancer drugs in the clinic that form covalent adducts on receptor and nonreceptor tyrosine kinases through Michael addition [reviewed in Zhao and Bourne (2018)]. Afatinib, osimertinib, and neratinib form irreversible adducts on cysteine residues in the catalytic sites of epidermal growth factor receptors for 
the treatment of non-small-cell lung cancers and breast cancers, whereas ibrutinib targets Bruton's tyrosine kinase for the treatment of lymphoma/leukemia. Additionally, there is renewed interest in targeting ERK1/2 with small molecules via allosteric inhibition of substrate interactions sites, such as the DRS (Sammons et al., 2019). Recent studies have identified a small-molecule inhibitor that covalently binds to a conserved cysteine (C159) in the DRS of ERK2 (Kaoud et al., 2019). This compound selectively blocked the activation of ERK1/2, did not modify other members of the MAP kinase family, and inhibited the proliferation of melanoma cells with BRAF (V600E) mutations that were resistant to BRAF inhibitors (Kaoud et al., 2019). Future studies will be aimed at identifying whether cysteine residues on other proteins may be modified by SF-3-030 to establish their role in cell signaling and growth inhibition.

The second objective of the studies was to evaluate global effects of SF-3-030 on gene and protein changes to gain insight into this chemical scaffold's mechanism of action. The transcriptome and proteome data show that the overall number of genes and proteins affected by the lead compound, SF-3-030, are less than or similar to the number of proteins targeted by known ERK1/2 or MEK1/2 ATP-competitive or catalytic site inhibitors. Similar to the ATP-competitive ERK1/2 pathway inhibitors, SF-3-030 appears to be more selective for cancer cells containing constitutively activating ERK1/2 pathway mutations. Although rapidly dividing cells are typically more sensitive to anticancer drugs, SF-3-030 effects on a select number of cells with activating ERK1/2 pathway mutations were shown to be independent of cell doubling times. Although further studies with additional cell lines will be needed to verify this observation, these data indicate that off-target effects on nontransformed cells may be limited.

Transcriptome and proteome analyses indicated that SF-3$\mathbf{0 3 0}$ induced rapid changes in the levels of ERK1/2-regulated transcription factors consistent with an oxidative stress response. Transcription factors such as c-Fos and c-Jun are elevated in response to oxidative or metabolic stress (Webster et al., 1994), and ROS-mediated induction of ERK1/2 can enhance Elk-1-mediated transcription and c-Fos expression (Müller et al., 1997). It was intriguing that SF-3-030 caused a robust induction of c-Fos but inhibited c-Myc protein levels (Fig. 3) and that these changes occurred at the level of transcription (Supplemental Table 2). Other examples in which protein levels increase because of SF-3-030 induction of transcription include c-Jun and HMOX1. In contrast, SF-3030 also inhibited Fra-1 levels, but there was no evidence this occurred through effects on transcription (Fig. 3; Supplemental Table 2). It is possible that the 1-hour time point used to evaluate transcriptome changes by RNA sequencing did not capture changes in Fra-1 transcription. The expression of Fra-1 and other Fos family proteins is regulated by c-Fos and subsequent AP-1 complex activity (Milde-Langosch, 2005). However, c-Fos induction in the current studies does not correlate with increased AP-1 activity, which we previously showed to be inhibited by SF-3-030 (Samadani et al., 2015; Defnet et al., 2019). Although apoptosis can be induced by c-Fos induction (Preston et al., 1996), further studies will be needed to clarify the requirement for c-Fos expression in SF-3-030mediated cell death in the absence of AP-1 activity.

ERK1/2 activation increases the expression of c-Myc (Kerkhoff et al., 1998), which can protect melanoma cells against oxidative stress (Benassi et al., 2006). In contrast, SF-3-030 mediated downregulation of c-Myc and its potential role in responding to elevated ROS by regulating the synthesis of antioxidants such as glutathione (Gao et al., 2009). After exogenous hydrogen peroxide-induced oxidative stress, ERK1/2 phosphorylation of c-Myc at serine 62 facilitates c-Myc recruitment to the GCLC promoter and the expression of GCLC, the rate-limiting enzyme involved in glutathione synthesis (Benassi et al., 2006). However, in the current studies, the dramatic inhibition of c-Myc protein levels by SF-3-030 after 8 hours (Fig. 3A) is not observed with GCLC (Supplemental Fig. 7). Nonetheless, targeted inhibition of c-Myc with ERK1/2 pathway inhibitors is a suggested approach to decrease the proliferation of melanoma cells and overcome drug resistance (Ciuffreda et al., 2009; Korkut et al., 2015).

The role of ROS in promoting or inhibiting cancer cells is subject to debate, and there is evidence to support that either increased or decreased ROS production can sensitize cancer cells to growth inhibition and cell death (Trachootham et al., 2009; Galadari et al., 2017). Although the use of antioxidant strategies may protect against some cancers (Trachootham et al., 2009), there are limited clinical data that support the beneficial effects of using antioxidant supplements to reduce the risk of developing cancer (Goodman et al., 2011). However, the mechanisms used by many anticancer drugs to kill cells involves increased oxidative stress (Trachootham et al., 2009). Melanocytes and melanoma cells are particularly sensitive to ROS, and the ROS dose will likely determine whether cells are able to adapt or will die (Meierjohann, 2014). Additional evidence supports the use of oxidative stress to inhibit melanoma cell metastasis (Piskounova et al., 2015). Although ROS activation may not be sufficient to inhibit all cancer cells, enhanced ROS production may also play a role in improving anticancer efficacy of combination therapies (Adams et al., 2013). Recent data suggest that therapeutic approaches that increased ROS may be beneficial to patients who have developed resistance to BRAF and MEK1/2 inhibitors (Wang et al., 2018).

The selectivity of SF-3-030 toward cancer cells with activating ERK1/2 pathway mutations supports the idea that exploiting ROS homeostasis may be therapeutically beneficial in some cancers. Although upregulation of ROS may provide a survival advantage in melanomas that are resistant to ERK1/2 pathway inhibitors, additional increases in ROS with histone deacetylase inhibitors promoted an apoptotic response in these cells (Wang et al., 2018). Further studies will be needed to determine whether SF-3-030 induces DNA instability and ROS production like histone deacetylase inhibitors. DNA damage may induce the ROS-responsive tumor suppressor OSGIN1 protein to enhance apoptosis in osteosarcoma cells through a mechanism involving p53 disruption of mitochondria functions and cytochrome c release ( $\mathrm{Hu}$ et al., 2012). Although SF-3-030 induced the protein levels of OSGIN1 and a subset of NRF2-regulated genes (Fig. 6, A and B), the NRF2 inhibitor brusatol did not affect SF-3-030 inhibition of A375 cell proliferation (Fig. 6F). These findings raise the potential to develop ROS activators that are lethal to cancer cells but do not promote an NRF2 response that leads to chemoresistance and cancer cell survival (Okazaki et al., 2020). Taken together, the current studies provide mechanistic support to evaluate how ROS production by $\mathbf{S F - 3 - 0 3 0}$ and 
related compounds can be used as an approach to inhibit the proliferation of cancers cells with activating mutations in the ERK1/2 pathway.

\section{Acknowledgments}

We thank Jacob Scheenstra for technical assistance.

\section{Authorship Contributions}

Participated in research design: Martinez, Huang, Samadani, Kane, Fletcher, Shapiro.

Conducted experiments: Martinez, Huang, Samadani, Centola, Chen, Hom.

Contributed new reagents or analytic tools: Chen, Conlon, Fletcher.

Performed data analysis: Martinez, Huang, Samadani, Mackowiak, Centola, Hom, Shapiro.

Wrote or contributed to the writing of the manuscript: Martinez, Huang, Hom, Kane, Fletcher, Shapiro.

\section{References}

Abel EV, Basile KJ, Kugel CH III, Witkiewicz AK, Le K, Amaravadi RK, Karakousis GC, Xu X, Xu W, Schuchter LM, et al. (2013) Melanoma adapts to RAF/MEK inhibitors through FOXD3-mediated upregulation of ERBB3. J Clin Invest 123: $2155-2168$

Adams DJ, Boskovic ZV, Theriault JR, Wang AJ, Stern AM, Wagner BK, Shamji AF, and Schreiber SL (2013) Discovery of small-molecule enhancers of reactive oxygen species that are nontoxic or cause genotype-selective cell death. ACS Chem Biol 8 923-929.

Alonso SR, Ortiz P, Pollán M, Pérez-Gómez B, Sánchez L, Acuña MJ, Pajares R, Martínez-Tello FJ, Hortelano CM, Piris MA, et al. (2004) Progression in cutaneous malignant melanoma is associated with distinct expression profiles: a tissue microarray-based study. Am J Pathol 164:193-203.

Anders S, McCarthy DJ, Chen Y, Okoniewski M, Smyth GK, Huber W, and Robinson MD (2013) Count-based differential expression analysis of RNA sequencing data using R and Bioconductor. Nat Protoc 8:1765-1786.

Arora A and Scholar EM (2005) Role of tyrosine kinase inhibitors in cancer therapy. $J$ Pharmacol Exp Ther 315:971-979.

Arvind R, Shimamoto H, Momose F, Amagasa T, Omura K, and Tsuchida N (2005) A mutation in the common docking domain of ERK2 in a human cancer cell line, which was associated with its constitutive phosphorylation. Int $J$ Oncol $\mathbf{2 7}$ $1499-1504$

Benassi B, Fanciulli M, Fiorentino F, Porrello A, Chiorino G, Loda M, Zupi G, and Biroccio A (2006) c-Myc phosphorylation is required for cellular response to oxidative stress. Mol Cell 21:509-519.

Bennasroune A, Gardin A, Aunis D, Crémel G, and Hubert P (2004) Tyrosine kinase receptors as attractive targets of cancer therapy. Crit Rev Oncol Hematol 50:23-38.

Boston SR, Deshmukh R, Strome S, Priyakumar UD, MacKerell AD Jr, and Shapiro $\mathrm{P}$ (2011) Characterization of ERK docking domain inhibitors that induce apoptosis by targeting Rsk-1 and caspase-9. BMC Cancer 11:7.

Burkhard KA, Chen F, and Shapiro P (2011) Quantitative analysis of ERK2 interactions with substrate proteins: roles for kinase docking domains and activity in determining binding affinity. $J$ Biol Chem 286:2477-2485.

Carlton JG, Caballe A, Agromayor M, Kloc M, and Martin-Serrano J (2012) ESCRTIII governs the Aurora B-mediated abscission checkpoint through CHMP4C. Science 336:220-225.

Cesi G, Walbrecq G, Zimmer A, Kreis S, and Haan C (2017) ROS production induced by BRAF inhibitor treatment rewires metabolic processes affecting cell growth of melanoma cells. Mol Cancer 16:102.

Chen F, Hancock CN, Macias AT, Joh J, Still K, Zhong S, MacKerell AD Jr, and Shapiro P (2006) Characterization of ATP-independent ERK inhibitors identified through in silico analysis of the active ERK2 structure. Bioorg Med Chem Lett 16:6281-6287.

Ciuffreda L, Del Bufalo D, Desideri M, Di Sanza C, Stoppacciaro A, Ricciardi MR, Chiaretti S, Tavolaro S, Benassi B, Bellacosa A, et al. (2009) Growth-inhibitory and antiangiogenic activity of the MEK inhibitor PD0325901 in malignant melanoma with or without BRAF mutations. Neoplasia 11:720-731.

da Silva Almeida AC, Abate F, Khiabanian H, Martinez-Escala E, Guitart J, Tensen CP, Vermeer MH, Rabadan R, Ferrando A, and Palomero T (2015) The mutational landscape of cutaneous T cell lymphoma and Sézary syndrome. Nat Genet 47: $1465-1470$

Dayalan Naidu S and Dinkova-Kostova AT (2020) KEAP1, a cysteine-based sensor and a drug target for the prevention and treatment of chronic disease. Open Biol 10:200105

Defnet AE, Huang W, Polischak S, Yadav SK, Kane MA, Shapiro P, and Deshpande DA (2019) Effects of ATP-competitive and function-selective ERK inhibitors on airway smooth muscle cell proliferation. FASEB $J$ 33:10833-10843.

Dorfer V, Pichler P, Stranzl T, Stadlmann J, Taus T, Winkler S, and Mechtler K (2014) MS Amanda, a universal identification algorithm optimized for high accuracy tandem mass spectra. J Proteome Res 13:3679-3684.

Drobic B, Pérez-Cadahía B, Yu J, Kung SK, and Davie JR (2010) Promoter chromatin remodeling of immediate-early genes is mediated through $\mathrm{H} 3$ phosphorylation at either serine 28 or 10 by the MSK1 multi-protein complex. Nucleic Acids Res $\mathbf{3 8}$ 3196-3208.
Emery CM, Vijayendran KG, Zipser MC, Sawyer AM, Niu L, Kim JJ, Hatton C, Chopra R, Oberholzer PA, Karpova MB, et al. (2009) MEK1 mutations confer resistance to MEK and B-RAF inhibition. Proc Natl Acad Sci USA 106:20411-20416.

Eng JK, Fischer B, Grossmann J, and Maccoss MJ (2008) A fast SEQUEST cross correlation algorithm. J Proteome Res 7:4598-4602

Erde J, Loo RR, and Loo JA (2014) Enhanced FASP (eFASP) to increase proteome coverage and sample recovery for quantitative proteomic experiments. J Proteome Res 13:1885-1895.

Fedorenko IV, Gibney GT, Sondak VK, and Smalley KS (2015) Beyond BRAF: where next for melanoma therapy? Br J Cancer 112:217-226.

Flaherty KT and McArthur G (2010) BRAF, a target in melanoma: implications for solid tumor drug development. Cancer 116:4902-4913.

Galadari S, Rahman A, Pallichankandy S, and Thayyullathil F (2017) Reactive oxygen species and cancer paradox: to promote or to suppress? Free Radic Biol Med 104:144-164.

Gao P, Tchernyshyov I, Chang TC, Lee YS, Kita K, Ochi T, Zeller KI, De Marzo AM, Van Eyk JE, Mendell JT, et al. (2009) c-Myc suppression of miR-23a/b enhances mitochondrial glutaminase expression and glutamine metabolism. Nature 458: 762-765.

Germann UA, Furey BF, Markland W, Hoover RR, Aronov AM, Roix JJ, Hale M, Boucher DM, Sorrell DA, Martinez-Botella G, et al. (2017) Targeting the MAPK signaling pathway in cancer: promising preclinical activity with the novel selective ERK1/2 inhibitor BVD-523 (ulixertinib). Mol Cancer Ther 16:2351-2363

Goodman M, Bostick RM, Kucuk O, and Jones DP (2011) Clinical trials of antioxidants as cancer prevention agents: past, present, and future. Free Radic Biol Med 51:1068-1084.

Guan C, He L, Chang Z, Gu X, Liang J, and Liu R (2020) ZNF774 is a potent suppressor of hepatocarcinogenesis through dampening the NOTCH2 signaling. Oncogene 39:1665-1680.

Hancock CN, Macias A, Lee EK, Yu SY, Mackerell AD Jr, and Shapiro P (2005) Identification of novel extracellular signal-regulated kinase docking domain inhibitors. J Med Chem 48:4586-4595.

Haq R, Shoag J, Andreu-Perez P, Yokoyama S, Edelman H, Rowe GC, Frederick DT, Hurley AD, Nellore A, Kung AL, et al. (2013) Oncogenic BRAF regulates oxidative metabolism via PGC1 $\alpha$ and MITF. Cancer Cell 23:302-315.

Benjamini Y and Hochberg Y (1995) Controlling the false discovery rate: a practical and powerful approach to multiple testing. J R Stat Soc Series B Stat Methodol 57: $289-300$

Hu J, Yao H, Gan F, Tokarski A, and Wang Y (2012) Interaction of OKL38 and p53 in regulating mitochondrial structure and function. PLoS One 7:e43362.

Johannessen CM, Boehm JS, Kim SY, Thomas SR, Wardwell L, Johnson LA, Emery CM, Stransky N, Cogdill AP, Barretina J, et al. (2010) COT drives resistance to RAF inhibition through MAP kinase pathway reactivation. Nature 468:968-972.

Käll L, Canterbury JD, Weston J, Noble WS, and MacCoss MJ (2007) Semisupervised learning for peptide identification from shotgun proteomics datasets. Nat Methods 4:923-925.

Kaoud TS, Johnson WH, Ebelt ND, Piserchio A, Zamora-Olivares D, Van Ravenstein SX, Pridgen JR, Edupuganti R, Sammons R, Cano M, et al. (2019) Modulating multi-functional ERK complexes by covalent targeting of a recruitment site in vivo. Nat Commun 10:5232

Kerkhoff E, Houben R, Löffler S, Troppmair J, Lee JE, and Rapp UR (1998) Regulation of c-myc expression by Ras/Raf signalling. Oncogene 16:211-216.

Korkut A, Wang W, Demir E, Aksoy BA, Jing X, Molinelli EJ, Babur Ö, Bemis DL, Onur Sumer S, Solit DB, et al. (2015) Perturbation biology nominates upstreamdownstream drug combinations in RAF inhibitor resistant melanoma cells. eLife 4 : e04640.

Krämer A, Green J, Pollard J Jr, and Tugendreich S (2014) Causal analysis approaches in ingenuity pathway analysis. Bioinformatics 30:523-530.

Kunz M, Moeller S, Koczan D, Lorenz P, Wenger RH, Glocker MO, Thiesen HJ, Gross G, and Ibrahim SM (2003) Mechanisms of hypoxic gene regulation of angiogenesis factor Cyr61 in melanoma cells. J Biol Chem 278:45651-45660.

Lee S, Warthaka M, Yan C, Kaoud TS, Ren P, and Dalby KN (2011) Examining docking interactions on ERK2 with modular peptide substrates. Biochemistry 50: $9500-9510$

Li R, Chen W, Yanes R, Lee S, and Berliner JA (2007) OKL38 is an oxidative stress response gene stimulated by oxidized phospholipids. J Lipid Res 48:709-715.

MacDonald G, Nalvarte I, Smirnova T, Vecchi M, Aceto N, Dolemeyer A, Frei A, Lienhard S, Wyckoff J, Hess D, et al. (2014) Memo is a copper-dependent redox protein with an essential role in migration and metastasis. Sci Signal 7:ra56.

Madan E, Gogna R, Kuppusamy P, Bhatt M, Mahdi AA, and Pati U (2013) SCO2 induces p53-mediated apoptosis by Thr845 phosphorylation of ASK-1 and dissociation of the ASK-1-Trx complex. Mol Cell Biol 33:1285-1302.

Mahalingam M, Arvind R, Ida H, Murugan AK, Yamaguchi M, and Tsuchida N (2008) ERK2 CD domain mutation from a human cancer cell line enhanced anchorage-independent cell growth and abnormality in Drosophila. Oncol Rep 20 957-962

Maik-Rachline G and Seger R (2016) The ERK cascade inhibitors: towards overcoming resistance. Drug Resist Updat 25:1-12.

Meierjohann S (2014) Oxidative stress in melanocyte senescence and melanoma transformation. Eur J Cell Biol 93:36-41.

Mendelsohn J and Baselga J (2006) Epidermal growth factor receptor targeting in cancer. Semin Oncol 33:369-385.

Mi H, Huang X, Muruganujan A, Tang H, Mills C, Kang D, and Thomas PD (2017) PANTHER version 11: expanded annotation data from Gene Ontology and Reactome pathways, and data analysis tool enhancements. Nucleic Acids Res 45: D183-D189.

Milde-Langosch K (2005) The Fos family of transcription factors and their role in tumourigenesis. Eur J Cancer 41:2449-2461.

Morris EJ, Jha S, Restaino CR, Dayananth P, Zhu H, Cooper A, Carr D, Deng Y, Jin W, Black S, et al. (2013) Discovery of a novel ERK inhibitor with activity in models of acquired resistance to BRAF and MEK inhibitors. Cancer Discov 3:742-750. 
Müller JM, Cahill MA, Rupec RA, Baeuerle PA, and Nordheim A (1997) Antioxidants as well as oxidants activate c-fos via Ras-dependent activation of extracellularsignal-regulated kinase 2 and Elk-1. Eur J Biochem 244:45-52.

Okazaki K, Papagiannakopoulos T, and Motohashi H (2020) Metabolic features of cancer cells in NRF2 addiction status. Biophys Rev 12:435-441.

Olayanju A, Copple IM, Bryan HK, Edge GT, Sison RL, Wong MW, Lai ZQ, Lin ZX, Dunn K, Sanderson CM, et al. (2015) Brusatol provokes a rapid and transient inhibition of Nrf2 signaling and sensitizes mammalian cells to chemical toxicity-implications for therapeutic targeting of Nrf2. Free Radic Biol Med 78:202-212.

Piskounova E, Agathocleous M, Murphy MM, Hu Z, Huddlestun SE, Zhao Z, Leitch AM, Johnson TM, DeBerardinis RJ, and Morrison SJ (2015) Oxidative stress inhibits distant metastasis by human melanoma cells. Nature 527:186-191.

Polsky D and Cordon-Cardo C (2003) Oncogenes in melanoma. Oncogene 22: 3087-3091.

Poulikakos PI and Rosen N (2011) Mutant BRAF melanomas--dependence and resistance. Cancer Cell 19:11-15.

Poulikakos PI and Solit DB (2011) Resistance to MEK inhibitors: should we co-target upstream? Sci Signal 4:pe16.

Pratilas CA, Taylor BS, Ye Q, Viale A, Sander C, Solit DB, and Rosen N (2009) (V600E)BRAF is associated with disabled feedback inhibition of RAF-MEK sig naling and elevated transcriptional output of the pathway. Proc Natl Acad Sci USA 106:4519-4524.

Preston GA, Lyon TT, Yin Y, Lang JE, Solomon G, Annab L, Srinivasan DG, Alcorta DA, and Barrett JC (1996) Induction of apoptosis by c-Fos protein. Mol Cell Biol 16: 211-218.

Qi D, Brownridge P, Xia D, Mackay K, Gonzalez-Galarza FF, Kenyani J, Harman V, Beynon RJ, and Jones AR (2012) A software toolkit and interface for performing stable isotope labeling and top3 quantification using Progenesis LC-MS. OMICS 16:489-495.

Ren D, Villeneuve NF, Jiang T, Wu T, Lau A, Toppin HA, and Zhang DD (2011) Brusatol enhances the efficacy of chemotherapy by inhibiting the Nrf2-mediated defense mechanism. Proc Natl Acad Sci USA 108:1433-1438.

Reuter CW, Morgan MA, and Bergmann L (2000) Targeting the Ras signaling pathway: a rational, mechanism-based treatment for hematologic malignancies? Blood 96:1655-1669.

Samadani R, Zhang J, Brophy A, Oashi T, Priyakumar UD, Raman EP, St John FJ, Jung KY, Fletcher S, Pozharski E, et al. (2015) Small-molecule inhibitors of ERK mediated immediate early gene expression and proliferation of melanoma cells expressing mutated BRaf. Biochem J 467:425-438.

Sammons RM, Perry NA, Li Y, Cho EJ, Piserchio A, Zamora-Olivares DP, Ghose R, Kaoud TS, Debevec G, Bartholomeusz C, et al. (2019) A novel class of common docking domain inhibitors that prevent ERK2 activation and substrate phosphorylation. ACS Chem Biol 14:1183-1194.

Santini R, Pietrobono S, Pandolfi S, Montagnani V, D’Amico M, Penachioni JY, Vinci MC, Borgognoni L, and Stecca B (2014) SOX2 regulates selfrenewal and tumorigenicity of human melanoma-initiating cells. Oncogene 33:4697-4708.

Sassone-Corsi P, Mizzen CA, Cheung P, Crosio C, Monaco L, Jacquot S, Hanauer A, and Allis CD (1999) Requirement of Rsk-2 for epidermal growth factor-activated phosphorylation of histone H3. Science 285:886-891.

Shah NG, Tulapurkar ME, Ramarathnam A, Brophy A, Martinez R III, Hom K, Hodges T, Samadani R, Singh IS, MacKerell AD Jr, et al. (2017) Novel noncatalytic substrate-selective p38 $\alpha$-specific MAPK inhibitors with endothelial-stabilizing and anti-inflammatory activity. J Immunol 198:3296-3306.

Shapiro P (2002) Ras-MAP kinase signaling pathways and control of cell proliferation: relevance to cancer therapy. Crit Rev Clin Lab Sci 39:285-330.

Shaw PE and Saxton J (2003) Ternary complex factors: prime nuclear targets for mitogen-activated protein kinases. Int J Biochem Cell Biol 35:1210-1226.
Shin SY, Bahk YY, Ko J, Chung IY, Lee YS, Downward J, Eibel H, Sharma PM, Olefsky JM, Kim YH, et al. (2006) Suppression of Egr-1 transcription through targeting of the serum response factor by oncogenic H-Ras. EMBO J 25:1093-1103.

Singh A, Venkannagari S, Oh KH, Zhang YQ, Rohde JM, Liu L, Nimmagadda S, Sudini K, Brimacombe KR, Gajghate S, et al. (2016) Small molecule inhibitor of NRF2 selectively intervenes therapeutic resistance in KEAP1-deficient NSCLC tumors. ACS Chem Biol 11:3214-3225.

Singh J, Petter RC, Baillie TA, and Whitty A (2011) The resurgence of covalent drugs. Nat Rev Drug Discov 10:307-317.

Sunryd JC, Cheon B, Graham JB, Giorda KM, Fissore RA, and Hebert DN (2014) TMTC1 and TMTC2 are novel endoplasmic reticulum tetratricopeptide repeatcontaining adapter proteins involved in calcium homeostasis. $J$ Biol Chem 289 : 16085-16099

Taus T, Köcher T, Pichler P, Paschke C, Schmidt A, Henrich C, and Mechtler K (2011) Universal and confident phosphorylation site localization using phosphoRS. $J$ Proteome Res 10:5354-5362.

Telkoparan-Akillilar P, Suzen S, and Saso L (2019) Pharmacological applications of Nrf2 inhibitors as potential antineoplastic drugs. Int J Mol Sci 20:2025.

Trachootham D, Alexandre J, and Huang P (2009) Targeting cancer cells by ROSmediated mechanisms: a radical therapeutic approach? Nat Rev Drug Discov 8: 579-591.

Tuma RS (2011) Getting around PLX4032: studies turn up unusual mechanisms of resistance to melanoma drug. J Natl Cancer Inst 103:170-171, 177.

von Kriegsheim A, Baiocchi D, Birtwistle M, Sumpton D, Bienvenut W, Morrice N, Yamada K, Lamond A, Kalna G, Orton R, et al. (2009) Cell fate decisions are specified by the dynamic ERK interactome. Nat Cell Biol 11:1458-1464.

Wang L, Leite de Oliveira R, Huijberts S, Bosdriesz E, Pencheva N, Brunen D, Bosma A, Song J-Y, Zevenhoven J, Los-de Vries GT, et al. (2018) An acquired vulnerability of drug-resistant melanoma with therapeutic potential. Cell 173:1413-1425.e14.

Webster KA, Discher DJ, and Bishopric NH (1994) Regulation of fos and jun immediate-early genes by redox or metabolic stress in cardiac myocytes. Circ Res 74:679-686.

Williamson JC, Edwards AV, Verano-Braga T, Schwämmle V, Kjeldsen F, Jensen ON, and Larsen MR (2016) High-performance hybrid Orbitrap mass spectrometers for quantitative proteome analysis: observations and implications. Proteomics 16: 907-914.

Wiśniewski JR, Zougman A, Nagaraj N, and Mann M (2009) Universal sample preparation method for proteome analysis. Nat Methods 6:359-362.

Wu P, Nielsen TE, and Clausen MH (2015) FDA-approved small-molecule kinase inhibitors. Trends Pharmacol Sci 36:422-439.

Wu P, Nielsen TE, and Clausen MH (2016) Small-molecule kinase inhibitors: an analysis of FDA-approved drugs. Drug Discov Today 21:5-10.

Yeh TC, Marsh V, Bernat BA, Ballard J, Colwell H, Evans RJ, Parry J, Smith D, Brandhuber BJ, Gross S, et al. (2007) Biological characterization of ARRY-142886 (AZD6244), a potent, highly selective mitogen-activated protein kinase kinase 1/2 inhibitor. Clin Cancer Res 13:1576-1583.

Yuan L, Mishra R, Patel H, Alanazi S, Wei X, Ma Z, and Garrett JT (2020) BRAF mutant melanoma adjusts to BRAF/MEK inhibitors via dependence on increased antioxidant SOD2 and increased reactive oxygen species levels. Cancers (Basel) 12:1661.

Zhao Z and Bourne PE (2018) Progress with covalent small-molecule kinase inhibitors. Drug Discov Today 23:727-735.

Zhong $\mathrm{H}$ and Bowen JP (2011) Recent advances in small molecule inhibitors of VEGFR and EGFR signaling pathways. Curr Top Med Chem 11:1571-1590.

Address correspondence to: Paul Shapiro, Department of Pharmaceutical Sciences, University of Maryland-Baltimore School of Pharmacy, 20 Penn St., Room 561, Baltimore, MD 21201. E-mail: pshapiro@rx.umaryland.edu 